\title{
One-dimensional Electric Field Structure of an Outer Gap Accelerator - III. Location of the Gap and the Gamma-ray Spectrum
}

\author{
K. Hirotani ${ }^{1}$ and S. Shibata ${ }^{2}$ \\ 1 National Astronomical Observatory, Osawa, Mitaka, Tokyo 181-8588, Japan Email: hirotani@hotaka.mtk.nao.ac.jp \\ ${ }^{2}$ Department of Physics, Yamagata University, Yamagata 990-8560, Japan Email: shibata@sci.kj.yamagata-u.ac.jp
}

Accepted . Received 2000 November; in original form 2000 November

\begin{abstract}
We investigate a stationary particle acceleration zone in the outer magnetosphere of a spinning neutron star. The charge depletion due to a global current causes a large electric field along the magnetic field lines. Migratory electrons and/or positrons are accelerated by this field to radiate curvature gamma-rays, some of which collide with the $\mathrm{X}$-rays to materialize as pairs in the gap. As a result of this pair-production cascade, the replenished charges partially screen the electric field, which is self-consistently solved together with the distribution of particles and gamma-rays. If no current is injected at neither of the boundaries of the accelerator, the gap is located around the so-called null surface, where the local Goldreich-Julian charge density vanishes. However, we find that the gap position shifts outwards (or inwards) when particles are injected at the inner (or outer) boundary. We apply the theory to the nine pulsars of which X-ray fields are known from observations. We show that the gap should be located near to or outside of the null surface for the Vela pulsar and PSR B1951+32, so that their expected $\mathrm{GeV}$ spectrum may be consistent with observations. We then demonstrate that the intrinsically large TeV flux from the outer gap of PSR B0540-69 is absorbed by the magnetospheric infrared photons to be undetectable. We also point out that the electrodynamic structure and the resultant $\mathrm{GeV}$ emission properties of millisecond pulsars are similar to young pulsars.
\end{abstract}

Key words: gamma-rays: observation - gamma-rays: theory - magnetic field - pulsars: individual (B0540-69, B1055-52, B1509-58, B1951+32, Geminga, J0437-4715, J0822-4300, J1617-5055, Vela)

\section{INTRODUCTION}

The EGRET experiment on the Compton Gamma Ray Observatory has detected pulsed signals from seven rotationpowered pulsars (for Crab, Nolan et al. 1993, Fierro et al. 1998; for Vela, Kanbach et al. 1994, Fierro et al. 1998; for Geminga, Mayer-Hassel Wander et al. 1994, Fierro et al. 1998; for PSR B1706-44, Thompson et al. 1996; for PSR B1046-58, Kaspi at al. 2000; for PSR B1055-52, Thompson et al. 1999; for PSR B1951+32, Ramanamurthy et al. 1995). The modulation of the $\gamma$-ray light curves at GeV energies testifies to the production of $\gamma$-ray radiation in the pulsar magnetospheres either at the polar cap (Harding, Tademaru, \& Esposito 1978; Daugherty \& Harding 1982, 1996; Dermer \& Sturner 1994; Sturner, Dermer, \& Michel 1995; Shibata, Miyazaki, \& Takahara 1998), or at the vacuum gaps in the outer magnetosphere (Cheng, Ho, \& Ruderman 1986a,b, hereafter CHR; Chiang \& Romani 1992,
1994; Romani and Yadigaroglu 1995; Romani 1996; Zhang \& Cheng 1997, hereafter ZC97). Effective $\gamma$-ray production in a pulsar magnetosphere may be extended to the very high energy (VHE) region above $100 \mathrm{GeV}$ as well; however, the predictions of fluxes by the current models of $\gamma$-ray pulsars are not sufficiently conclusive (e.g., Cheng 1994). Whether or not the spectra of $\gamma$-ray pulsars continue up to the VHE region is a question which remains one of the interesting issues of high-energy astrophysics.

In the VHE region, positive detections of radiation at a high confidence level have been reported from the direction of the Crab, B1706-44, and Vela pulsars (Nel et al. 1993; Edwards et al. 1994; Yoshikoshi et al. 1997; see also Kifune 1996 for a review), by virtue of the technique of imaging Cerenkov light from extensive air showers. However, as for pulsed $\mathrm{TeV}$ radiation, only the upper limits have been, as a rule, obtained from these pulsars (see the references cited just above). If the VHE emission originates the pulsar mag- 
netosphere, rather than the extended nebula, a significant fraction of them can be expected to show pulsation. Therefore, the lack of pulsed $\mathrm{TeV}$ emissions provides a severe constraint on the modeling of particle acceleration zones in a pulsar magnetosphere.

In fact, in CHR picture, the magnetosphere should be optically thick for pair-production in order to reduce the $\mathrm{TeV}$ flux to an unobserved level by absorption. This in turn requires very high luminosities of tertiary photons in the infrared energy range. However, the required fluxes are generally orders of magnitude larger than the observed values (Usov 1994). We are therefore motivated by the need to contrive an outer-gap model which produces less $\mathrm{TeV}$ emission with a moderate infrared luminosity.

High-energy emission from a pulsar magnetosphere, in fact, crucially depends on the acceleration electric field, $E_{\|}$, along the magnetic field lines. It was Hirotani and Shibata (1999a,b,c; hereafter Papers I, II, III), and Hirotani (2000a,b,c; hereafter Papers IV, V, VI) who first solved the spatial distribution of $E_{\|}$together with particle and $\gamma$-ray distribution functions. They considered a pair-production cascade in a neutron star magnetosphere by solving the Vlasov equations (see also Beskin et al. 1992).

By this method, they explicitly solved the gap width along the field lines, $E_{\|}$, particle densities, and the $\gamma$-ray distribution functions. They further demonstrated that a stationary gap is formed around the null surface at which the local Goldreich-Julian charge density,

$\rho_{\mathrm{GJ}}=\frac{\Omega B_{z}}{2 \pi c}$,

vanishes (fig. 1), where $B_{z}$ is the component of the magnetic field along the rotation axis, $\Omega$ refers to the angular frequency of the neutron star, and $c$ is the speed of light. Equation (1) is valid unless the gap is located close to the light cylinder, of which distance from the rotation axis is given by

$\varpi_{\mathrm{LC}}=3 \times 10^{8} \Omega_{2}{ }^{-1} \mathrm{~cm}$,

where $\Omega_{2} \equiv \Omega /\left(100 \mathrm{rad} \mathrm{s}^{-1}\right)$. In this paper, we develop the method presented in Paper VI as follows:

- We compute the curvature radius, $R_{\mathrm{C}}$, at each point along the magnetic field line, rather than assuming $R_{\mathrm{C}}=0.5 \varpi_{\mathrm{LC}}$ throughout the gap.

- We partially take the effect of the unsaturated particles into account (eq. 38).

- We investigate the gap structure when particles flow into the gap from the inner or the outer boundaries.

- We compute the explicit spectra of $\mathrm{TeV}$ emission due to inverse Compton scatterings, rather than estimating only their upper limits.

In the next two sections, we describe the physical processes of pair production cascade in the outer magnetosphere of a pulsar. We then apply the theory to individual pulsars and present the expected $\mathrm{GeV}$ and $\mathrm{TeV}$ spectra for various boundary conditions on the current densities in $\S$. In the final section, we compare the results with previous works.

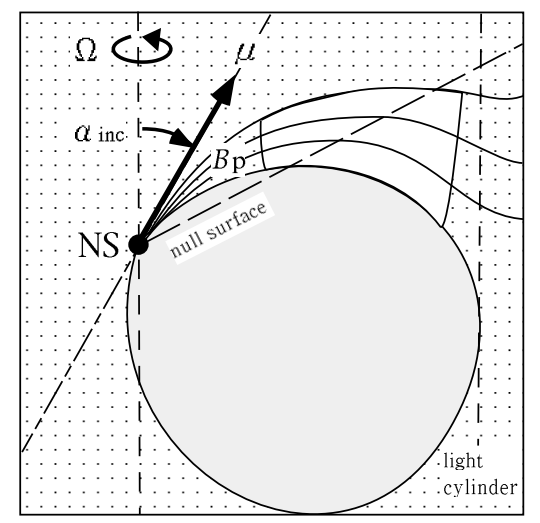

Figure 1. Side view of a hypothetical outer-magnetospheric gap. On the null surface, the Goldreich-Julian charge density vanishes.

\section{BASIC EQUATIONS AND BOUNDARY CONDITIONS}

We first reduce the Poisson equation for the electric potential into a one-dimensional form in $\S 2.1$. We then give the Boltzmann equations of the particles and the $\gamma$-rays in $\S \S 2.2$ and 2.3, and describe the Lorentz factors and the X-ray fields in $\S \S 2.4$ and 2.5. We finally impose suitable boundary conditions in $\S 2.6$.

\subsection{Reduction of the Poisson Equation}

In the Poisson equation for the electrostatic potential $\Phi$, the special relativistic effects appear in the higher orders of $(\Omega \varpi / c)^{2}$, where $\varpi$ indicates the distance of the point from the rotational axis (Shibata 1995). As the first-order approximation, we neglect such terms. To simplify the geometry, we further introduce a rectilinear approximation. Unless the gap width, $W$, along the field lines becomes a good fraction of $\varpi_{L C}$, we can approximate the magnetic field lines as straight lines parallel to the $s$-axis (figure 2). Here, $s$ is an outwardly increasing coordinate along a magnetic field line, while $y$ designates the azimuth. Assuming that typical transfield thickness of the gap, $D_{\perp}$, is greater than $W$, we can Fourier analyze in the transfield direction to rewrite the Poisson equation into (eq. [3] in Paper VI)

$$
-\frac{d^{2}}{d s^{2}} \Phi=-\frac{\Phi}{D_{\perp}^{2}}+4 \pi e\left(N_{+}-N_{-}-\frac{\rho_{\mathrm{GJ}}}{e}\right),
$$

where $N_{+}$and $N_{-}$refer to the positronic and electronic densities, respectively, $e$ the magnitude of the charge on the electron, and $s$ the length along the last-open fieldline. In what follows, we define the origin of $s$ as the neutron star surface.

It is convenient to non-dimensionalize the length scales by a typical Debey scale length $c / \omega_{\mathrm{p}}$, where

$\omega_{\mathrm{p}}=\sqrt{\frac{4 \pi e^{2}}{m_{\mathrm{e}}} \frac{\Omega B_{\mathrm{cnt}}}{2 \pi c e}}=1.875 \times 10^{7} \Omega_{2}{ }^{1 / 2} B_{5}{ }^{1 / 2} \mathrm{rad} \mathrm{s}^{-1}$;

$B_{5}$ represents the magnetic field strength at the gap center in $10^{5} \mathrm{G}$ unit. The dimensionless coordinate variable then becomes 


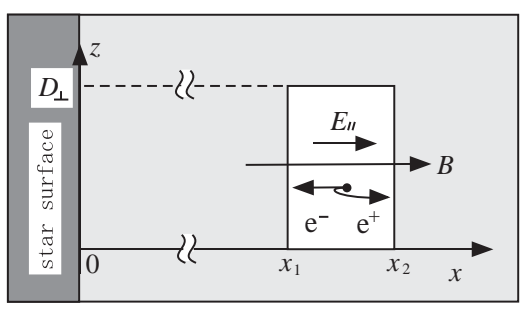

Figure 2. Rectilinear approximation to the outer gap. The $y$-axis, which designates the azimuth, is not depicted to avoid complication. This approximation is valid unless the gap width, $W=s_{2}-s_{1}$, becomes a good fraction of $\varpi_{\mathrm{LC}}$.

$\xi \equiv\left(\omega_{\mathrm{p}} / c\right) s=6.25 \times 10^{-4} \Omega_{2}{ }^{1 / 2} B_{5}{ }^{1 / 2} s$.

By using such dimensionless quantities, we can rewrite the Poisson equation into

$E_{\|}=-\frac{d \varphi}{d \xi}$,

$\frac{d E_{\|}}{d \xi}=-\frac{\varphi}{\Delta_{\perp}^{2}}+\frac{B(\xi)}{B_{\mathrm{cnt}}}\left[n_{+}(\xi)-n_{-}(\xi)\right]+\frac{B_{z}(\xi)}{B_{\mathrm{cnt}}}$

where $\Delta_{\perp} \equiv\left(\omega_{\mathrm{p}} / c\right) D_{\perp}$ and $\varphi(\xi) \equiv e \Phi(x) /\left(m_{\mathrm{e}} c^{2}\right)$; the particle densities are normalized in terms of a typical value of the Goldreich-Julian density as

$n_{ \pm}(\xi) \equiv \frac{2 \pi c e}{\Omega} \frac{N_{ \pm}(x)}{B(x)}$.

$B_{\text {cnt }} \equiv B\left(\xi_{\text {cnt }}\right)$ refers to the magnetic field strength at the gap center, $\xi=\xi_{\mathrm{cnt}}$. In the non-relativistic limit, the local Goldreich-Julian charge density is given by

$\frac{B_{z}(\xi)}{B_{\mathrm{cnt}}}=\frac{2 \cos \theta \cos \left(\theta-\alpha_{\mathrm{i}}\right)-\sin \theta \sin \left(\theta-\alpha_{\mathrm{i}}\right)}{\sqrt{1+3 \cos ^{2} \theta_{\mathrm{cnt}}}}$,

where $\alpha_{\mathrm{i}}$ refers to the inclination angle of the magnetic moment, $\theta$ and $\theta_{\text {cnt }}$ the colatitude angle of the position at which $\rho_{\mathrm{GJ}}$ is measured and that at the gap center, respectively. Neglecting the general relativistic effect near to the star surface, we can implicitly solve $\theta$ in terms of $s$ from

$\frac{s}{\varpi_{\mathrm{LC}}}=\int_{\theta_{*}}^{\theta} \frac{\sin \left(\theta-\alpha_{\mathrm{i}}\right) \sqrt{1+3 \cos ^{2}\left(\theta-\alpha_{\mathrm{i}}\right)}}{\sin \theta_{\mathrm{LC}} \sin ^{2}\left(\theta_{\mathrm{LC}}-\alpha_{\mathrm{i}}\right)} d \theta$,

where $\theta_{*}$ is the colatitude angle of the last-open fieldline at the star surface; it is given by

$\frac{\sin ^{2}\left(\theta_{*}-\alpha_{\mathrm{i}}\right)}{r_{*}}=\frac{\sin ^{2}\left(\theta_{\mathrm{LC}}-\alpha_{\mathrm{i}}\right)}{\varpi_{\mathrm{LC}} / \sin \theta_{\mathrm{LC}}}$,

where $r_{*}$ refers to the neutron-star radius, and $\theta_{\mathrm{LC}}$ the colatitude angle of the intersection of the last-open fieldline and the light cylinder. We can solve $\theta_{\mathrm{LC}}$ for a given $\alpha_{\mathrm{i}}$ from

$2 \cot \left(\theta_{\mathrm{LC}}-\alpha_{\mathrm{i}}\right) \sin \theta_{\mathrm{LC}}+\cos \theta_{\mathrm{LC}}=0$.

\subsection{Particle Continuity Equation}

Let us now consider the continuity equations of the particles. It should be noted that almost all the particles migrate with the large, saturated Lorentz factor. We therefore assume here that both electrostatic and curvature-radiation- reaction forces cancel out each other to obtain the following continuity equations

$$
\pm B \frac{d}{d s}\left(\frac{N_{ \pm}}{B}\right)=\frac{1}{c} \int_{0}^{\infty} d \epsilon_{\gamma}\left[\eta_{\mathrm{p}+} G_{+}+\eta_{\mathrm{p}-} G_{-}\right],
$$

where $G_{ \pm}\left(x, \epsilon_{\gamma}\right)$ are the distribution function of $\gamma$-ray photons having momentum $\pm m_{\mathrm{e}} c \epsilon_{\gamma}$ along the poloidal field line. Since the electric field is assumed to be positive in the gap, $e^{+}$'s (or $e^{-}$'s) migrate outwards (or inwards). The pair production redistribution function for outwardly (or inwardly) propagating $\gamma$-rays are written as $\eta_{\mathrm{p}+}\left(\right.$ or $\left.\eta_{\mathrm{p}-}\right)$ and are defined by

$\eta_{\mathrm{p} \pm}\left(\epsilon_{\gamma}\right)=\left(1-\mu_{\mathrm{c}}\right) \frac{c}{\omega_{\mathrm{p}}} \int_{\epsilon_{\mathrm{th}}}^{\infty} d \epsilon_{\mathrm{x}} \frac{d N_{\mathrm{x}}}{d \epsilon_{\mathrm{x}}} \sigma_{\mathrm{p}}\left(\epsilon_{\gamma}, \epsilon_{\mathrm{x}}, \mu_{\mathrm{c}}\right)$,

where $\sigma_{\mathrm{p}}$ is the pair-production cross section and $\cos ^{-1} \mu_{\mathrm{c}}$ refers to the collision angle between outwardly (or inwardly) propagating $\gamma$-rays and the X-ray photon with energy $m_{\mathrm{e}} c^{2} \epsilon_{\mathrm{x}}$; X-ray number density between dimensionless energies $\epsilon_{\mathrm{x}}$ and $\epsilon_{\mathrm{x}}+d \epsilon_{\mathrm{x}}$, is integrated over $\epsilon_{\mathrm{x}}$ from the threshold energy $\epsilon_{\mathrm{th}} \equiv 2\left(1-\mu_{\mathrm{c}}\right)^{-1} \epsilon_{\gamma}^{-1}$ to the infinity. The explicit expression of $\sigma_{\mathrm{p}}$ is given by (Berestetskii et al. 1989)

$$
\begin{aligned}
& \sigma_{\mathrm{p}}\left(\epsilon_{\gamma}, \epsilon_{\mathrm{x}}, \mu_{\mathrm{c}}\right) \\
& \equiv \frac{3}{16} \sigma_{\mathrm{T}}\left(1-v^{2}\right)\left[\left(3-v^{4}\right) \ln \frac{1+v}{1-v}-2 v\left(2-v^{2}\right)\right], \\
& v\left(\epsilon_{\gamma}, \epsilon_{\mathrm{x}}, \mu_{\mathrm{c}}\right) \equiv \sqrt{1-\frac{2}{1-\mu_{\mathrm{c}}} \frac{1}{\epsilon_{\gamma} \epsilon_{\mathrm{x}}}},
\end{aligned}
$$

where $\sigma_{\mathrm{T}}$ is the Thomson cross section, and $\epsilon_{\mathrm{x}} \equiv E_{\mathrm{x}} / m_{\mathrm{e}} c^{2}$ refers to the dimensionless energy of an X-ray photon.

To evaluate $\mu_{\mathrm{c}}$ between the surface X-rays and the $\gamma$ rays, we must consider $\gamma$-ray's toroidal momenta due to an aberration of light. It should be noted that a $\gamma$-ray photon propagates in the instantaneous direction of the particle motion at the time of the emission. The toroidal velocity of a particle at the gap center $\left(r_{\mathrm{cnt}}, \theta_{\mathrm{cnt}}\right)$ becomes

$v_{\phi}=r_{\mathrm{cnt}} \sin \theta_{\mathrm{cnt}} \Omega+\kappa B_{\phi}-E_{\|} B_{\phi} \frac{c}{B^{2}}$,

where $\kappa$ is a constant. On the right-hand side, the first term is due to corotation, while second one due to magnetic bending. Since $E_{\|}$arises in the gap, the corresponding drift velocity appears as the third term. Unless the gap is located close to the light cylinder, we can neglect the terms containing $B_{\phi}$ as a first-order approximation. We thus have $v_{\phi}=r_{\mathrm{cnt}} \sin \theta_{\mathrm{cnt}} \Omega$. Since the three-dimensional particle velocity is virtually $c$, the angle between the particle motion and the poloidal plane becomes

$\phi_{\mathrm{ab}}=\sin ^{-1}\left(\frac{r_{\mathrm{cnt}} \sin \theta_{\mathrm{cnt}} \Omega}{c}\right)=\sin ^{-1}\left(\frac{r_{\mathrm{cnt}} \sin \theta_{\mathrm{cnt}}}{\varpi_{\mathrm{LC}}}\right)$.

We evaluate $\left(r_{\mathrm{cnt}}, \theta_{\mathrm{cnt}}\right)$ along a Newtonian dipole. Using $\phi_{\mathrm{ab}}$, we can compute $\mu_{\mathrm{c}}$ as

$\mu_{\mathrm{c}}=\cos \phi_{\mathrm{ab}} \cos \theta_{\mathrm{pol}}$,

where $\theta_{\text {pol }}$ is the collision angle between the surface X-rays and the curvature $\gamma$-rays, that is, the angle between the two vectors $\left(r_{\mathrm{cnt}}, \theta_{\mathrm{cnt}}\right)$ and $\left(B^{r}, B^{\theta}\right)$ at the gap center. Therefore, the collision approaches head-on (or tail-on) for inwardly (or outwardly) propagating $\gamma$-rays, as the gap shifts towards the star.

For magnetospheric, power-law X-rays, as assume 
$\mu_{\mathrm{c}}=\cos \left(W / \varpi_{\mathrm{LC}}\right)$,

for both inwardly and outwardly propagating $\gamma$-rays. Aberration of light is not important for this component, because both the X-rays and the $\gamma$-rays are emitted nearly at the same place, provided that $W \ll \varpi_{\mathrm{LC}}$ holds.

Let us introduce the following dimensionless $\gamma$-ray densities in the dimensionless energy interval between $\beta_{i-1}$ and $\beta_{i}$ such that

$g_{ \pm}^{i}(\xi) \equiv \frac{2 \pi c e}{\Omega B_{\mathrm{cnt}}} \int_{\beta_{i-1}}^{\beta_{i}} d \epsilon_{\gamma} G_{ \pm}\left(x, \epsilon_{\gamma}\right)$.

In this paper, we set $\beta_{0}=10^{2}$; this implies that the lowest $\gamma$-ray energy considered is $m_{\mathrm{e}} c^{2}=51.1 \mathrm{MeV}$. To cover a wide range of $\gamma$-ray energies, we divide the $\gamma$-ray spectra into 9 energy bins and put $\beta_{1}=3.162 \times 10^{2}, \beta_{2}=10^{3}, \beta_{3}=$ $3.162 \times 10^{3}, \beta_{4}=10^{4}, \beta_{5}=3.162 \times 10^{4}, \beta_{6}=5.623 \times 10^{4}$, $\beta_{7}=10^{5} . \beta_{8}=1.778 \times 10^{5}, \beta_{9}=3.162 \times 10^{5}$.

We can now rewrite equation 13 into the dimensionless form,

$\frac{d n_{ \pm}}{d \xi}= \pm \frac{B_{\mathrm{cnt}}}{B(\xi)} \sum_{i=1}^{9}\left[\eta_{\mathrm{p}+}{ }^{i} g_{+}^{i}(\xi)+\eta_{\mathrm{p}-}{ }^{i} g_{-}^{i}(\xi)\right]$

where $\eta_{\mathrm{p} \pm}^{i}$ are evaluated at the central energy in each bin as

$\eta_{\mathrm{p} \pm}^{i} \equiv \frac{1}{\omega_{\mathrm{p}}} \eta_{\mathrm{p} \pm}\left(\frac{\beta_{i-1}+\beta_{i}}{2}\right)$.

A combination of equations (21) gives the current conservation law,

$j_{\text {tot }} \equiv n_{+}(\xi)+n_{-}(\xi)=$ constant for $\xi$.

When $j_{\text {tot }}=1.0$, the current density per unit flux tube equals the Goldreich-Julian value, $\Omega /(2 \pi)$.

\subsection{Gamma-Ray Boltzmann Equations}

Let us next consider the $\gamma$-ray Boltzmann equations. The $\gamma$ rays are beamed in the direction of the magnetic field where it was emitted. Therefore, the propagation direction of each $\gamma$-ray photon does not coincide with the local magnetic field where the $\gamma$-ray distribution is evaluated. However, to avoid complications, we simply assume that the outwardly (or inwardly) propagating $\gamma$-rays dilate (or constrict) at the same rate with the magnetic field (Paper VII). Then we can write down the $\gamma$-ray Boltzmann equations into the form,

$\pm B \frac{d}{d s}\left[\frac{1}{B} G_{ \pm}\left(x, \epsilon_{\gamma}\right)\right]=-\frac{1}{c} \eta_{\mathrm{p} \pm} G_{ \pm}\left(x, \epsilon_{\gamma}\right)+\frac{1}{c} \eta_{\mathrm{c}} N_{ \pm}(x)$,

where (e.g., Rybicki, Lightman 1979)

$\eta_{\mathrm{c}} \equiv \frac{\sqrt{3} e^{2} \Gamma}{h R_{\mathrm{C}}} \frac{1}{\epsilon_{\gamma}} F\left(\frac{\epsilon_{\gamma}}{\epsilon_{\mathrm{c}}}\right)$

$\epsilon_{\mathrm{c}} \equiv \frac{1}{m_{\mathrm{e}} c^{2}} \frac{3}{4 \pi} \frac{h c \Gamma^{3}}{R_{\mathrm{C}}}$

$F(s) \equiv s \int_{x}^{\infty} K_{\frac{5}{3}}(t) d t$

$R_{\mathrm{C}}$ is the curvature radius of the magnetic field lines and $K_{5 / 3}$ is the modified Bessel function of $5 / 3$ order. The effect of the broad spectrum of curvature $\gamma$-rays is represented by the factor $F\left(\epsilon_{\gamma} / \epsilon_{\mathrm{c}}\right)$ in equation (25). In the polar coordinates $(r, \theta)$, the explicit expression of $R_{\mathrm{C}}$ is given by
$\frac{R_{\mathrm{C}}}{\varpi_{\mathrm{LC}}}=\left|1+\left(\frac{d \zeta}{d \xi}\right)^{2}\right|^{3 / 2} \times\left|\frac{d^{2} \zeta}{d \xi^{2}}\right|^{-1}$,

where

$\zeta \equiv r \cos \theta / \varpi_{\mathrm{LC}}$

$\xi \equiv r \sin \theta / \varpi_{\mathrm{LC}}$.

Assuming a Newtonian dipole magnetic field with inclination $\alpha_{\mathrm{i}}$, we can write the derivatives in equation(28) as

$\frac{d \zeta}{d \xi}=\frac{\cos \alpha_{\mathrm{i}}-(3 / 2) \sin \theta \sin \left(\theta-\alpha_{\mathrm{i}}\right)}{\sin \alpha_{\mathrm{i}}+(3 / 2) \cos \theta \sin \left(\theta-\alpha_{\mathrm{i}}\right)}$,

$\frac{d^{2} \zeta}{d \xi^{2}}=\frac{1}{\rho} \frac{(d \zeta / d \xi) f-\sin \theta \cos \alpha_{\mathrm{i}}+(3 / 2) \cos \theta \sin \alpha_{\mathrm{i}}}{\sin \alpha_{\mathrm{i}}+(3 / 2) \cos \theta \sin \left(\theta-\alpha_{\mathrm{i}}\right)}$,

where

$\frac{\sin ^{2}\left(\theta-\alpha_{\mathrm{i}}\right)}{\rho}=\frac{\sin ^{2}\left(\theta_{*}-\alpha_{\mathrm{i}}\right)}{r_{*} / \varpi_{\mathrm{LC}}}$,

$f \equiv\left(\cos \theta \sin \alpha_{\mathrm{i}}-\frac{3}{2} \sin \theta \cos \alpha_{\mathrm{i}}\right) \frac{d \zeta}{d \xi}+\frac{1}{2} \cos \left(\theta+\alpha_{\mathrm{i}}\right)$.

Integrating (24) in the energy intervals $\left[\beta_{i-1}, \beta_{i}\right]$, we obtain

$\pm \frac{d}{d \xi} g_{ \pm}{ }^{i}(\xi)= \pm \frac{d}{d \xi}(\ln B) g_{ \pm}^{i}-\eta_{\mathrm{p} \pm}^{i} g_{ \pm}^{i}+\eta_{\mathrm{c}}^{i} \frac{B(\xi)}{B_{\mathrm{cnt}}} n_{ \pm}$,

where $i=1,2, \cdots, m(m=9)$ and

$$
\begin{aligned}
\eta_{\mathrm{c}}^{i} \equiv & \frac{\sqrt{3} e^{2} \Gamma}{\omega_{\mathrm{p}} h R_{\mathrm{C}}} \int_{\beta_{i-1} / \epsilon_{\mathrm{c}}}^{\beta_{i} / \epsilon_{\mathrm{c}}} d u \int_{u}^{\infty} K_{\frac{5}{3}}(t) d t \\
= & 2.14 \times 10^{-8} \Gamma\left(\frac{R_{\mathrm{C}}}{0.5 \varpi_{\mathrm{LC}}}\right)^{-1} \sqrt{\frac{\Omega_{2}}{B_{5}}} \\
& \times \int_{\beta_{i-1} / \epsilon_{\mathrm{c}}}^{\beta_{i} / \epsilon_{\mathrm{c}}} d s \int_{s}^{\infty} K_{\frac{5}{3}}(t) d t .
\end{aligned}
$$

\subsection{Terminal Lorentz Factor}

The most effective assumption for particle motion in the gap arises from the fact that the velocity immediately saturates in the balance between the electric force and the radiation reaction force due to curvature radiation. Equating the electric force $e|d \Phi / d x|$ and the radiation reaction force, we obtain the saturated Lorentz factor at each point,

$\Gamma_{\text {sat }}=\left(\frac{3 R_{\mathrm{C}}^{2}}{2 e}\left|\frac{d \Phi}{d x}\right|+1\right)^{1 / 4}$.

When $W$ is so small that a significant fraction of the particles are unsaturated, equation (37) overestimates the particle's Lorentz factors. To take account of such unsaturated motion of the particles, we compute $\Gamma$ by

$\frac{1}{\Gamma}=\sqrt{\frac{1}{\Gamma_{\mathrm{sat}}{ }^{2}}+\frac{1}{\varphi^{2}\left(\xi_{2}\right)}}$,

where

$\varphi\left(\xi_{2}\right)=\frac{e \Phi\left(x_{2}\right)}{m_{\mathrm{e}} c^{2}}$

represents the maximum attainable Lorentz factor; the gap is considered to exists in $\xi_{1}<\xi<\xi_{2}$ and the potential origin is chosen such that $\varphi\left(\xi_{1}\right)=0$. 


\subsection{X-Ray Field}

To execute the integration in equation (14), we must specify the X-ray field illuminating the gap. X-ray field of a rotationpowered neutron star within the light cylinder can be attributed to the following three emission processes:

(1) Photospheric emission from the whole surface of a cooling neutron star (Greenstein \& Hartke 1983; Romani 1987; Shibanov et al. 1992; Pavlov et al. 1994; Zavlin et al. 1995). (2) Thermal emission from the neutron star's polar caps which are heated by the bombardment of relativistic particles streaming back to the surface from the magnetosphere (Kundt \& Schaaf 1993; Zavlin, Shibanov, \& Pavlov 1995; Gil \& Krawczyk 1996).

(3) Non-thermal emission from relativistic particles accelerated in the pulsar magnetosphere (Ochelkov \& Usov 1980a,b; El-Gowhari \& Arponen 1972; Aschenbach \& Brinkmann 1975; Hardee \& Rose 1974; Daishido 1975).

The spectrum of the first component is expected to be expressed with a modified blackbody. However, for simplicity, we approximate it in terms of a Plank function with temperature $k T_{\mathrm{s}}$, because the X-ray spectrum is occasionally fitted by a simple blackbody spectrum. We regard a blackbody component as the first one if its observed emitting area, $A_{\mathrm{s}}$, is comparable with the whole surface of a neutron star, $A_{*} \equiv 4 \pi r_{*}^{2}$, where $r_{*}$ denotes the neutron star radius. We take account of both the pulsed and the non-pulsed surface blackbody emission as this soft blackbody component. At distance $r$ from the center of the star, the X-ray density between energies $\epsilon_{\mathrm{x}}$ and $\epsilon_{\mathrm{x}}+d \epsilon_{\mathrm{x}}$ is given by the Planck law,

$\frac{d N_{\mathrm{x}}}{d \epsilon_{\mathrm{x}}}=\frac{1}{4 \pi^{2}}\left(\frac{m_{\mathrm{e}} c^{2}}{c \hbar}\right)^{3}\left(\frac{A_{\mathrm{s}}}{4 \pi r^{2}}\right) \frac{\epsilon_{\mathrm{x}}{ }^{2}}{\exp \left(\epsilon_{\mathrm{x}} / \Delta_{\mathrm{s}}\right)-1}$,

where $\Delta_{\mathrm{s}}$ is defined by

$\Delta_{\mathrm{s}} \equiv \frac{k T_{\mathrm{s}}}{m_{\mathrm{e}} c^{2}}$

$k T_{\mathrm{s}}$ refers to the soft blackbody temperature measured by a distant observer. Since the outer gap is located outside of the deep gravitational potential well of the neutron star, the photon energy there is essentially the same as what a distant observer measures.

As for the second component, we regard a blackbody component as the heated polar cap emission if its observed emitting area, $A_{\mathrm{h}}$, is much smaller than $A_{*}$. We approximate its spectrum by a Planck function. We take account of both the pulsed and the non-pulsed polar cap emission as this hard blackbody component. In the same manner as in the soft blackbody case, the spectrum at a radius $r$ becomes

$\frac{d N_{\mathrm{x}}}{d \epsilon_{\mathrm{x}}}=\frac{1}{4 \pi^{2}}\left(\frac{m_{\mathrm{e}} c^{2}}{c \hbar}\right)^{3}\left(\frac{A_{\mathrm{h}}}{4 \pi r^{2}}\right) \frac{\epsilon_{\mathrm{x}}{ }^{2}}{\exp \left(\epsilon_{\mathrm{x}} / \Delta_{\mathrm{s}}\right)-1}$,

where $\Delta_{\mathrm{h}}$ is related with the hard blackbody temperature, $k T_{\mathrm{h}}$, as

$\Delta_{\mathrm{h}} \equiv \frac{k T_{\mathrm{h}}}{m_{\mathrm{e}} c^{2}}$.

Unlike the first and the second components, a power-law component is usually dominated by a nebula emission. To get rid of the nebula emission, which illuminates the outer gap inefficiently, we adopt only the pulsed components of a power-law emission as the third component. We describe a magnetospheric component with the power law, $\frac{d N_{\mathrm{pl}}}{d \epsilon_{\mathrm{x}}}=N_{\mathrm{pl}} \epsilon_{\mathrm{x}}^{\alpha} \quad\left(\epsilon_{\min }<\epsilon_{\mathrm{x}}<\epsilon_{\max }\right)$.

The photon index $\alpha$ is typically between -2 and -1 for a pulsed, power-law X-ray component in hard X-ray band (e.g., Saito 1998). We assume $\epsilon_{\min }=0.1 \mathrm{keV} / 511 \mathrm{keV}$ and $\epsilon_{\max }=100 \mathrm{keV} / 511 \mathrm{keV}$ for homogeneous discussion.

\subsection{Boundary Conditions}

To solve the Vlasov equations (6), (7), (21), and (35), we must impose boundary conditions. The inner boundary is defined so that $E_{\|}$vanishes there. Therefore, we have

$E_{\|}\left(\xi_{1}\right)=0$.

It is noteworthy that condition (45) is consistent with the stability condition at the plasma-vacuum interface (KrausePolstorff \& Michel 1985a,b). At the inner (starward) boundary $\left(\xi=\xi_{1}\right)$, we impose (Paper VI)

$\varphi\left(\xi_{1}\right)=0$,

$g_{+}{ }^{i}\left(\xi_{1}\right)=0 \quad(i=1,2, \cdots, m)$,

where $m=9$. Since the particles may be created inside of the outer gap (i.e., at $\xi<\xi_{1}$ ), positrons may flow into the gap at $\xi=\xi_{1}$ as a part of the global current pattern in the magnetosphere. We thus denote the positronic current divided by ce per unit magnetic flux tube at $\xi=\xi_{1}$ as

$n_{+}\left(\xi_{1}\right)=j_{1}$.

This yields, with the help of the charge-conservation law (eq. 23]),

$n_{-}\left(\xi_{1}\right)=j_{\text {tot }}-j_{1}$.

At the outer boundary $\left(\xi=\xi_{2}\right)$, we impose

$E_{\|}\left(\xi_{2}\right)=0$,

$g_{-}{ }^{i}\left(\xi_{2}\right)=0 \quad(i=1,2, \cdots, m)$,

$n_{-}\left(\xi_{2}\right)=j_{2}$.

The current (divided by ce) created in the gap per unit flux tube can be expressed as

$j_{\text {gap }}=j_{\text {tot }}-j_{1}-j_{2}$.

We adopt $j_{\text {gap }}, j_{1}$, and $j_{2}$ as the free parameters in this paper.

We have totally $2 m+6$ boundary conditions $45-(52)$ for $2 m+4$ unknown functions $\Phi, E_{\|}, n_{+}, n_{-}, g_{+}{ }^{1}, g_{+}{ }^{2}$, $\cdots, g_{+}{ }^{m}, g_{-}{ }^{1}, g_{-}{ }^{2}, \cdots, g_{-}{ }^{m}$. Thus two extra boundary conditions must be compensated by making the positions of the boundaries $\xi_{1}$ and $\xi_{2}$ be free. The two free boundaries appear because $E_{\|}=0$ is imposed at both the boundaries and because $j_{\text {gap }}$ is externally imposed. In other words, the gap boundaries $\left(\xi_{1}\right.$ and $\left.\xi_{2}\right)$, and hence the position shifts if $j_{1}$ and/or $j_{2}$ varies.

\section{TEV SPECTRA}

In $\mathrm{TeV}$ energies inverse Compton (IC) scatterings of infrared (IR) photons off relativistic electrons and positrons $(\Gamma \sim$ $10^{7}$ ) are the process of $\gamma$-ray production. We briefly discuss the IR field in $\S 3.1$, and describe the intrinsic $\mathrm{TeV}$ emission 
from the gap in $\S 3.2$ and the extrinsic absorption due to magnetospheric IR field in $\S 3.3$.

\subsection{Infrared photon field}

Without a careful consideration of the synchrotron beaming, it is difficult to estimate the specific intensity of the IR field. Therefore, we simply assume that the IR field are homogeneous and isotropic within the radius $\varpi_{L C}$. This assumption comes from the following emission scenario in the magnetosphere: The primary $\gamma$-rays emitted in the gap collide with $\mathrm{X}$-ray and IR photons outside of the gap to materialize as primary pairs, which emit secondary photons in X-ray and soft $\gamma$-ray energies via synchrotron process. Some portions of the secondary photons collide with each other to materialize as tertiary pairs in the magnetosphere. The tertiary pairs have much lower energies compared with the secondary pairs and emit copious tertiary IR photons via synchrotron process. On these grounds, we may expect that the tertiary IR field has lost the directional information of the primary $\gamma$-rays, compared with the secondary X-ray field.

We do not adopt a broken power-law to describe the soft photons from IR to X-ray energies. This is because the adopted assumptions are different each other. As described in $\S 2.5$, we assume that the secondary, power-law X-ray field is homogeneous only within the radius of $r_{\mathrm{cnt}}$ around the gap and is highly beamed along the magnetic field at the gap cener so that the collision angles between the primary $\gamma$-rays and the secondary $\mathrm{X}$-rays in the gap are typically $W / \varpi_{\mathrm{LC}}$ radian (eq. [19]). On the other hand, we assume that the tertiary, power-law IR field is homogeneous and isotropic within the radius $\varpi_{\mathrm{LC}}$.

At distance $r$ from the center of the star, we adopt the following power-law IR spectra:

$\frac{d N_{\mathrm{IR}}}{d \epsilon_{\mathrm{IR}}}=N_{\mathrm{IR}}\left(\frac{d}{\mathrm{kpc}}\right)^{2}\left(\frac{r}{\varpi_{\mathrm{LC}}}\right)^{-2} \epsilon_{\mathrm{IR}}{ }^{\alpha}$,

where $\epsilon_{\mathrm{IR}} m_{\mathrm{e}} c^{2}$ refers to the IR photon energy, and $\epsilon_{\mathrm{IR}, \min }<$ $\epsilon<\epsilon_{\mathrm{IR}, \max }$. We adopt $\epsilon_{\mathrm{IR}, \min }=10^{-6}$ and $\epsilon_{\mathrm{IR}, \min }=10^{-2}$; the results do not depend on these cut-off energies very much.

\subsection{Inverse Compton Scatterings}

When an electron or a positron is migrating with Lorentz factor $\Gamma \gg 1$ in an isotropic photon field, it upscatters the soft photons to produce the following number spectrum of $\gamma$-rays (Blumenthal \& Gould 1970):

$$
\begin{aligned}
& \quad \frac{d^{2} N}{d t d \epsilon_{\gamma}}=\frac{3}{4} \sigma_{\mathrm{T}} \frac{c}{\Gamma^{2}} \frac{d N_{\mathrm{IR}}}{d \epsilon_{\mathrm{IR}}} \frac{d \epsilon_{\mathrm{IR}}}{\epsilon_{\mathrm{IR}}} \\
& \times\left[2 q \ln q+(1+2 q)(1-q)+\frac{(Q q)^{2}(1-q)}{2(1+Q q)}\right],
\end{aligned}
$$

where $Q \equiv 4 \epsilon_{\mathrm{IR}} \Gamma$ and $q \equiv \epsilon_{\gamma} / Q\left(\Gamma-\epsilon_{\gamma}\right)$; here, $\epsilon_{\gamma}$ refers to the energy of the upscattered photons in $m_{\mathrm{e}} c^{2}$ unit. Substituting equation (54), integrating $d^{2} N / d t d \epsilon_{\gamma}$ over $\epsilon_{\mathrm{IR}}$, and multiplying the $\gamma$-ray energy $\left(\epsilon_{\gamma} m_{\mathrm{e}} c^{2}\right)$ and the electron number $\left(N_{\mathrm{e}}\right)$ in the gap, we obtain the flux density of the upscattered photons as a function of $\epsilon_{\gamma}$.

\subsection{Absorption due to pair production}

As mentioned in $\S 3.1$, the IR field is assumed to be homogeneous and isotropic within radius $\varpi_{\mathrm{LC}}$. Outside of $\varpi_{\mathrm{LC}}$, both the IR photon density and the collision angles decrease; therefore, we neglect the pair production at $r>\varpi_{\mathrm{LC}}$ for simplicity. The optical depth then becomes

$\tau\left(\epsilon_{\gamma}\right)=L \int_{\epsilon_{\mathrm{IR}, \min }}^{\epsilon_{\mathrm{IR}, \max }} \frac{d N_{\mathrm{IR}}}{d \epsilon_{\mathrm{IR}}} \sigma_{\mathrm{p}}\left(\epsilon_{\mathrm{IR}}, \epsilon_{\gamma}, \mu_{\mathrm{c}}\right) d \epsilon_{\mathrm{IR}}$,

where $L$ refers to the path length. For homogeneous discussion, we assume $L=0.5 \varpi_{\mathrm{LC}}$ in this paper. As the first order approximation, we adopt $90^{\circ}$ as the collision angles in the isotropic IR field to substitute $\mu_{\mathrm{c}}=0$ in equation (56), where $\sigma_{\mathrm{p}}$ is defined by equation (15).

\section{APPLICATION TO INDIVIDUAL PULSARS}

In this section, we apply the theory to the nine rotationpowered pulsars of which X-ray field at the outer gap can be deduced from observations. The Crab pulsar was investigated in detail in Paper VII; therefore, we exclude this young pulsar in this paper. We first describe their X-ray and infrared fields in the next two subsections, and present the electric field distribution in $\S 4.3$ and the resultant $\mathrm{GeV}$ and $\mathrm{TeV}$ emissions from individual pulsars in $\S 4.4$.

\subsection{Input X-ray Field}

We present the observed X-ray properties of individual pulsars in order of spin-down luminosity, $\dot{E}_{\text {rot }}$ (table 1 ). We assume $\epsilon_{\min }=0.1 \mathrm{keV} / 511 \mathrm{keV}$ and $\epsilon_{\max }=100 \mathrm{keV} / 511 \mathrm{keV}$ for homogeneous discussion.

B0540-69 From ASCA observations in 2-10 keV band, its X-ray radiation is known to be well fitted by a powerlaw with $\alpha=-2.0$. The unabsorbed luminosity in this energy range is $1.3 \times 10^{36} \mathrm{erg} \mathrm{s}^{-1}$, which leads to $N_{\mathrm{pl}}=$ $9.0 \times 10^{9} d^{2}\left(r_{0} / \varpi_{\mathrm{LC}}\right)^{-2}$ (Saito 1998), where $d$ is the distance to the pulsar in $\mathrm{kpc}$.

B1509-58 From ASCA observations in 2-10 keV band, its pulsed emission can be fitted by a power-law with $\alpha=-1.1$. The unabsorbed flux in this energy range is $2.9 \times 10^{-11} \mathrm{erg} / \mathrm{cm}^{2} / \mathrm{s}$, which leads to $N_{\mathrm{pl}}=9.3 \times$ $10^{11} d^{2}\left(r_{0} / \varpi_{\mathrm{LC}}\right)^{-2}$.

J1617-5055 and J0822-4300 These two pulsars have resemble parameters such as $\Omega \sim 90 \mathrm{rad} \mathrm{s}^{-1}, \mu \sim$ $10^{30.6} \mathrm{G} \mathrm{cm}^{3}$, and characteristic age $\sim 8 \times 10^{3} \mathrm{yr}$. From the ASCA observations of J1617-5055 in 3.5-10 keV band, its pulsed emission subtracted the background and the steady components can be fitted by a power-law with $\alpha=-1.6$ (Torii et al. 1998). Adopting the distance to be $3.3 \mathrm{kpc}$ (Coswell et al. 1975), we can calculate its unabsorbed flux as $3.1 \times 10^{-12} \mathrm{erg} / \mathrm{cm}^{2} / \mathrm{s}$, which yields $N_{\mathrm{pl}}=$ $6.5 \times 10^{10} d^{2}\left(r_{0} / \varpi_{\mathrm{LC}}\right)^{-2}$. On the other hand, the distance of J0822-4300 was estimated from VLA observations as $d=2.2 \pm 0.3 \mathrm{kpc}$ (Reynoso et al. 1995). ROSAT observations revealed that the soft X-ray emission of this pulsar is consistent with a single-temperature blackbody model with $k T_{\mathrm{s}}=0.28 \pm 0.10 \mathrm{keV}$ and $A_{\mathrm{s}} \sim 0.04 A_{*}(d / 2.2)^{2}$ (Petre, Becker, \& Winkler 1996). 
Table 1. Input X-ray field

\begin{tabular}{|c|c|c|c|c|c|c|c|c|c|}
\hline pulsar & $\begin{array}{c}\text { distance } \\
\mathrm{kpc}\end{array}$ & $\begin{array}{c}\Omega \\
\operatorname{rad} s^{-1}\end{array}$ & $\begin{array}{c}\log _{10} \mu \\
\lg \left(\mathrm{G} \mathrm{cm}^{3}\right)\end{array}$ & $\begin{array}{l}k T_{\mathrm{s}} \\
\mathrm{eV}\end{array}$ & $A_{\mathrm{S}} / A_{*}$ & $\begin{array}{c}k T_{\mathrm{h}} \\
\mathrm{eV}\end{array}$ & $A_{\mathrm{h}} / A_{*}$ & $\begin{array}{c}N_{\mathrm{pl}} \\
\mathrm{cm}^{-3}\end{array}$ & $-\alpha$ \\
\hline B0540-69 & 49.4 & 124.7 & 31.00 & $\ldots$ & $\ldots$ & $\ldots$ & $\ldots$ & $10^{14.15}$ & 2.0 \\
\hline B1509-58 & 4.40 & 41.7 & 31.19 & $\ldots$ & $\ldots$ & $\ldots$ & $\ldots$ & $10^{14.04}$ & 1.1 \\
\hline $\mathrm{J} 1617-5055$ & 3.30 & 90.6 & 30.78 & $\ldots$ & $\ldots$ & $\ldots$ & $\ldots$ & $10^{12.64}$ & 1.6 \\
\hline J0822-4300 & 2.20 & 83.4 & 30.53 & 280 & 0.040 & $\ldots$ & $\ldots$ & $\ldots$ & $\ldots$ \\
\hline Vela & 0.50 & 61.3 & 30.53 & 150 & 0.066 & $\ldots$ & $\ldots$ & $\ldots$ & $\ldots$ \\
\hline B1951+32 & 2.5 & 159 & 29.68 & $\ldots$ & $\ldots$ & $\ldots$ & $\ldots$ & $10^{13.55}$ & 1.6 \\
\hline Geminga & 0.16 & 26.5 & 30.21 & 48 & 0.16 & $\ldots$ & $\ldots$ & $10^{5.00}$ & 1.6 \\
\hline B1055-52 & 1.53 & 31.9 & 30.03 & 68 & 7.3 & 320 & $10^{-3.64}$ & $\ldots$ & $\ldots$ \\
\hline J0437-4715 & 0.180 & 1092 & 26.50 & 22 & 0.16 & 95 & $10^{-3.28}$ & $\ldots$ & $\ldots$ \\
\hline
\end{tabular}

Vela From ROSAT observations in 0.06-2.4 keV, the spectrum of its point source (presumably the pulsar) emission is expressed by two components: Surface blackbody component with $k T_{\mathrm{s}}=150 \mathrm{eV}$ and $A_{\mathrm{s}}=0.066 A_{*}(d / 0.5)^{2}$, and a power-law component with $\alpha=-3.3$ (Ögelman et al. 1993). However, the latter component does not show pulsations; therefore, we consider only the former component as the Xray field illuminating the outer gap.

B1951+32 From ROSAT observations in 0.1-2.4 keV, the spectrum of its point source (presumably the pulsar) emission can be fitted by a single power-law component with $\alpha=$ -1.6 and intrinsic luminosity of $2.3 \times 10^{33}(d / 2.5)^{2} \mathrm{erg} \mathrm{s}^{-1}$ (Safi-Harb \& Ögelman 1995), which yields $N_{\mathrm{pl}}=9.1 \times$ $10^{11} d^{2}\left(r_{0} / \varpi_{\mathrm{LC}}\right)^{-2}$. The extension of this power-law is consistent with the upper limit of the pulsed component in 2-10 keV energy band (Saito 1998).

Geminga The X-ray spectrum consists of two components: the soft surface blackbody with $k T_{\mathrm{s}}=50 \mathrm{eV}$ and $A_{\mathrm{s}}=0.22 A_{*}(d / 0.16)^{2}$ and a hard power law with $\alpha=-1.6$ and $N_{\mathrm{pl}}=3.9 \times 10^{6} d^{2}$ (Halpern \& Wang 1997). A parallax distance of 160pc was estimated from HST observations (Caraveo et al. 1996).

B1055-52 Combining ROSAT and ASCA data, Greiveldinger et al. (1996) reported that the X-ray spectrum consists of two components: a soft blackbody with $k T_{\mathrm{s}}=68$ $\mathrm{eV}$ and $A_{\mathrm{s}}=7.3 A_{*}(d / 1.53)^{2}$ and a hard blackbody with $k T_{\mathrm{h}}=320 \mathrm{eV}$ and $A_{\mathrm{h}}=2.3 \times 10^{-4} A_{*}(d / 1.53)^{2}$.

J0437-4715 Using ROSAT and EUVE data (Becker \& Trümper 1993; Halpern et at. 1996), Zavlin and Pavlov (1998) demonstrated that both the spectra and the light curves of its soft X-ray radiation can originate from hot polar caps with a nonuniform temperature distribution and be modeled by a step-like functions having two different temperatures. The first component is the emission from heated polar-cap core with temperature $k T_{\mathrm{h}}=10^{6.16} \mathrm{~K}$ measured at the surface and with an area $A_{\mathrm{h}}=5.3 \times$ $10^{-4} A_{*}(d / 0.180)^{2}$. The second one can be interpreted as a cooler rim around the polar cap on the neutron star surface with temperature $k T_{\mathrm{s}}=10^{5.53} \mathrm{~K}$ and with an area $A_{\mathrm{s}}=0.16 A_{*}(d / 0.180)^{2}$. Considering the gravitational redshift factor of 0.76 , the best-fit temperatures observed at infinity become $k T_{\mathrm{s}}=22 \mathrm{eV}$ and $k T_{\mathrm{h}}=95 \mathrm{eV}$. From parallax measurements, its distance is reported to be $178 \pm 26 \mathrm{pc}$ (Sandhu et al. 1997). We adopt $d=180 \mathrm{pc}$ as a representative value.

\subsection{Input Infrared Field}

We next consider the homogeneous and isotropic infrared field in the magnetosphere. In addition to the references cited below, see also Thompson et al. (1999).

B0540-69 Its de-extincted optical and soft X-ray pulsed flux densities can be interpolated as $F_{\nu}=0.207 \nu^{-0.3}$ Jy (Middleditch \& Pennypacker 1985). This line extrapolates to $0.47 \mathrm{mJy}$ at $640 \mathrm{MHz}$, which is consistent with an observed value $0.4 \mathrm{mJy}$ (Manchester et al. 1993). We thus extrapolates the relation $F_{\nu}=0.207 \nu^{-0.3}$ to the infrared energies and obtain (Paper V)

$d N_{\mathrm{IR}} / d \epsilon_{\mathrm{IR}}=1.6 \times 10^{12} d^{2} \epsilon_{\mathrm{IR}}{ }^{-1.3}$

B1509-58 The flux densities emitted from close to the neutron star in radio (Taylor, Manchester, \& Lyne 1993), optical (Caraveo, Mereghetti, \& Bignami 1994), soft X-ray (Seward et al. 1984), and hard X-ray (Kawai et al. 1993) bands can be fitted by a single power-law $F_{\nu}=1.36 \nu^{-0.32}$ Jy. We thus adopt

$d N_{\mathrm{IR}} / d \epsilon_{\mathrm{IR}}=4.7 \times 10^{11} d^{2} \epsilon_{\mathrm{IR}}{ }^{-1.3}$

Vela The flux densities emitted from close to the neutron star in radio (Taylor, Manchester, \& Lyne 1993; Downs, Reichley, \& Morris 1973), optical (Manchester et al. 1980) bands can be fitted by $F_{\nu}=1.71 \times 10^{7} \nu^{-0.91} \mathrm{Jy}$. We thus adopt

$d N_{\mathrm{IR}} / d \epsilon_{\mathrm{IR}}=1.8 \times 10^{7} d^{2} \epsilon_{\mathrm{IR}}{ }^{-1.9}$

B1951+32, The flux densities emitted from close to the neutron star in radio (Taylor, Manchester, \& Lyne 1993) and soft X-ray (Safi-Harb \& Ögelman, \& Finley 1995) bands can be interpolated as $F_{\nu}=32.8 \nu^{-0.49} \mathrm{Jy}$. We thus adopt

$d N_{\mathrm{IR}} / d \epsilon_{\mathrm{IR}}=6.3 \times 10^{10} d^{2} \epsilon_{\mathrm{IR}}{ }^{-1.5}$

Geminga The upper limit of the flux density in radio band (Taylor, Manchester, \& Lyne 1993) and the flux density in optical band (Shearer et al. 1998) gives spectral index greater (or harder) than -0.69 . Interpolating the infrared flux with these two frequencies, we obtain

$d N_{\mathrm{IR}} / d \epsilon_{\mathrm{IR}}=1.9 \times 10^{7} d^{2} \epsilon_{\mathrm{IR}}{ }^{-1.7}$,

which gives a conservative upper limit of infrared photon number density under the assumption of a single power-law interpolation.

B1055-52 The flux densities emitted from close to the neutron star in radio (Taylor, Manchester, \& Lyne 1993) 
and optical (Mignani et al. 1997) bands can be interpolated as $F_{\nu}=4.2 \times 10^{5} \nu^{-0.77} \mathrm{Jy}$. We thus adopt

$d N_{\mathrm{IR}} / d \epsilon_{\mathrm{IR}}=7.7 \times 10^{7} d^{2} \epsilon_{\mathrm{IR}}{ }^{-1.8}$,

J1617-5055, J0822-4300, and J0437-4715 There have been no available infrarad or optical observations for these three pulsars. We thus simply assume that $\alpha=-1.5$ for these four pulsars and that $\nu F_{\nu}=10^{9} \mathrm{Jy} \cdot \mathrm{Hz}$ at $0.01 \mathrm{eV}$. We then obtain

$d N_{\mathrm{IR}} / d \epsilon_{\mathrm{IR}}=2.5 \times 10^{7} d^{2} \epsilon_{\mathrm{IR}}^{-1.5}$

for J1617-5055,

$d N_{\mathrm{IR}} / d \epsilon_{\mathrm{IR}}=2.1 \times 10^{7} d^{2} \epsilon_{\mathrm{IR}}{ }^{-1.5}$

for J0822-4300, and

$d N_{\mathrm{IR}} / d \epsilon_{\mathrm{IR}}=3.6 \times 10^{13} d^{2} \epsilon_{\mathrm{IR}}^{-1.5}$

for J0437-4715.

\subsection{Electric Field Structure}

To examine the behavior of the solutions in a wide parameter space, we consider the following four cases: $\left(j_{1}, j_{2}\right)=(0,0)$, $(0.3,0),(0.6,0)$, and $(0,0.3)$. In what follows, we denote them as cases $1,2,3$, and 4, respectively. In case 1, particles flow into the gap at neither the inner nor the outer boundary. In case 2 (or case 4), the positronic (or electronic) current flowing into the gap per unit flux tube at the outer (or inner) boundary is $30 \%$ of the typical Goldreich-Julian value, $\Omega / 2 \pi$.

In case 1 , for $\alpha_{i}=45^{\circ}$, the solution for B1509-58 disappears if $j_{\text {gap }}>0.0825$ and that for B1055-52 disappears if $j_{\text {gap }}>0.0152$. This is because the electric field distribution forms a 'brim' (e.g., fig. 2 in Hirotani \& Okamoto 1998) as $j_{\text {gap }}$ approaches a certain upper limit, typically several percent of $\Omega / 2 \pi$. On these grounds, we fix $j_{\text {gap }}=0.01$ for all the four cases. We consider B1509-58, B1055-52, and J0437-4715 to describe $E_{\|}$distributions.

\subsubsection{B1509-58, a young pulsar}

Since the Crab pulsar was investigated in Paper VII, we consider here B1509-58 as a typical example of young pulsars. The results of $E_{\|}(s)$ for $\alpha_{\mathrm{i}}=45^{\circ}$ is presented in figure 3 . The solid, dashed, dash-dotted, and dotted lines correspond to the cases $1,2,3$, and 4 , respectively. The abscissa designates the distance along the last-open field line and covers the range from the neutron star surface $(s=0)$ to the position where the disance equals $s=1.2 \times \varpi_{\mathrm{LC}}=9.34 \times 10^{6} \mathrm{~m}$.

The solid line shows that the gap is located around the null surface, if no currents penetrate into the gap at neither of the boundaries. The gap slightly extends outwards, because the pair production mean free path becomes small at the outer region of the gap, owing to the diluted X-ray field there. It is noteworthy that the potential drop becomes the largest when $j_{1}=0$ and $j_{2}=0$ (i.e., case 1 ).

The dashed and dash-dotted lines indicate three points we may notice. For one thing, the gap shifts outwards as $j_{1}$ increases. This conclusion is consistent with the results obtained analytically by Shibata and Hirotani (2000). What is more, the maximum value of $E_{\|}$decreases as the gap shifts outwards. This is because the decreased $\rho_{\mathrm{GJ}}$ at larger distance reduces $\left|d E_{\|} / d s\right|$ (eq. [3]). One final point is that $W$ increases as the gap shifts outwards. This is because the pair production mean free path increases, owing to the diluted $\mathrm{X}$-ray field at large radii. The potential drop in the gap remains almost unchanged between cases 2 and 3 , because the decrease of maximum $E_{\|}$and the increase of $W$ cancel each other.

On the other hand, when $j_{2}$ increases, the gap shifts inwards. When the gap is located on the half way from the null surface to the star (case 4, the dotted line), the potential drop becomes only $3 \%$ of that in case 1 . A physical interpretation on this point is given in $\S 5.2$.

\subsubsection{PSR B1055-52, a middle-aged pulsar}

We next consider PSR B1055-52 as an example of middleaged pulsars. The $E_{\|}$distribution for $\alpha_{\mathrm{i}}=45^{\circ}$ is presented in figure 4 . The abscissa, ordinate, and the lines and the same as figure 3 .

Comparing with figure 3, we can understand that the gap is more extended for B1055-52. This is because the Xray field, which is supplied by the surface emission, becomes less dense for middle-aged pulsars. The weak X-ray field enlarges the pair-production mean free path, and hence the gap width. The strength of $E_{\|}$decreases compared with young pulsars, because local $\rho_{\mathrm{GJ}}$ is small due to their large $\varpi_{\mathrm{LC}}$, or equivalently, due to their small $\Omega$.

The position of the gap behaves in the same way as B1509-58. That is, it shifts towards the light cylinder (or the star surface) when $j_{1}$ (or $j_{2}$ ) increases.

\subsubsection{J0437-4715, a millisecond pulsar}

We finally consider J0437-4715, a millisecond pulsar. The $E_{\|}$distribution for $\alpha_{\mathrm{i}}=45^{\circ}$ is presented in figure 5. Even though the magnetic moment is small, the strength of $E_{\|}$is comparable with the young pulsar B1509-58. This is because the magnetic field in the outer magnetosphere is comparable with those of young pulsars, owing to its fast rotation $\left(\Omega=1092 \mathrm{rad} \mathrm{s}^{-1}\right)$, which reduces $\varpi_{\mathrm{LC}}$ to $2.74 \times 10^{5} \mathrm{~m}$. In addition, $W$ is much less than B1055-52, a middle-aged pulsar and relatively close to B1509-69, a young pulsar. This is because the shrunk light cylinder of millisecond pulsars results in a dense X-ray field illuminating the gap; the dense $\mathrm{X}$-ray field in turn reduces the pair-production mean free path, and hence $W$. This result is consistent with the semianalytical prediction for the millisecond pulsar B1821-24 (Paper V), of which X-ray field is dominated by a powerlaw component. In short, the electrodynamic structures of the gap are similar between millisecond pulsars and young pulsars.

\subsection{Gamma-ray Spectra}

The spectra of outwardly (or inwardly) propagating $\gamma$-rays in $\mathrm{GeV}$ energies, are readily computed from $g_{+}{ }^{i}\left(\xi_{2}\right)$ (or $\left.g_{-}{ }^{i}\left(\xi_{1}\right)\right)$. The TeV spectra, on the other hand, can be obtained by the method described in $\S$ 3. Except for B0540-69, the optical depth (eq. [56.) is much less than unity; therefore, the effect of absorption due to $\mathrm{TeV}-\mathrm{eV}$ collisions in the 


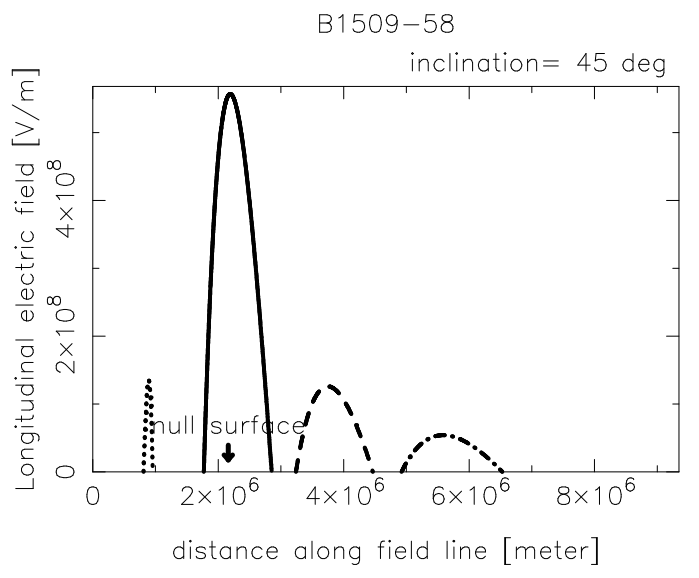

Figure 3. Distribution of the acceleration field in the magnetosphere of B1509-58, a young pulsar. The inclination angle is $45^{\circ}$. The abscissa is the distance from the star surface along the lastopen fieldline. The solid, dashed, dash-dotted, and dotted lines correspond to the cases $1,2,3$, and 4 , respectively (see text).

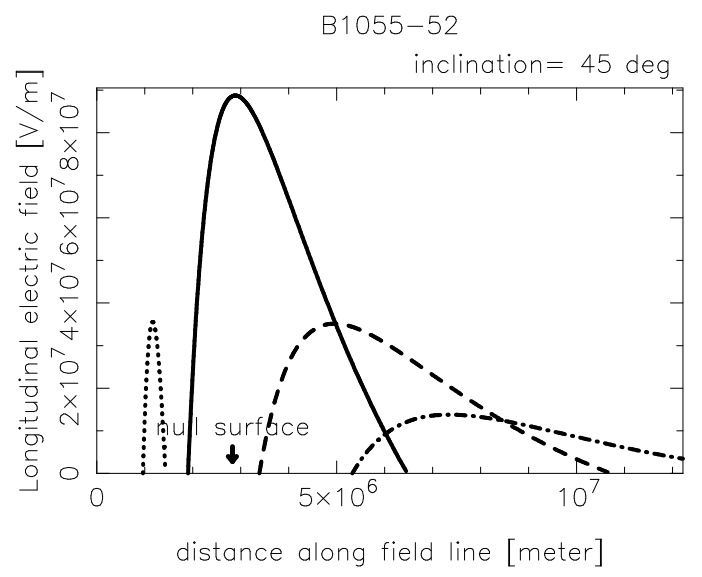

Figure 4. Distribution of the acceleration field for B1055-52, a middle-aged pulsar. The inclination angle is $45^{\circ}$. The abscissa, ordinate, and the lines are the same as figure figure 3 .

magnetosphere, can be neglected for the other eight pulsars considered in this paper.

We present the combined $\mathrm{GeV}-\mathrm{TeV}$ spectra for B054069, B1509-58, J1617-5055, J0822-4300, Vela, B1951+32, Geminga, B1055-52, and J0437-4715. in figures 8 18, multiplying the same cross sectional area for the $\mathrm{GeV}$ and $\mathrm{TeV}$ emissions for each pulsar. Both the inclination angle and $D_{\perp}$ are indicated at the top right corner. The outward (or inward) $\gamma$-ray flux is depicted by thick (or thin) lines. The observed pulsed fluxes and their upper limits are indicated by open circles (EGRET), open squares (Whipple), open triangles (Durham group). The upper limits of the stationary fluxes are obtained by CANGAROO group and are denoted by filled circles. To compare the results with the same $\alpha_{\mathrm{i}}$, we present the case of $\alpha_{\mathrm{i}}=45^{\circ}$ for all the pulsars. For Vela, B1951+32, Geminga, and B1055-52, we also present

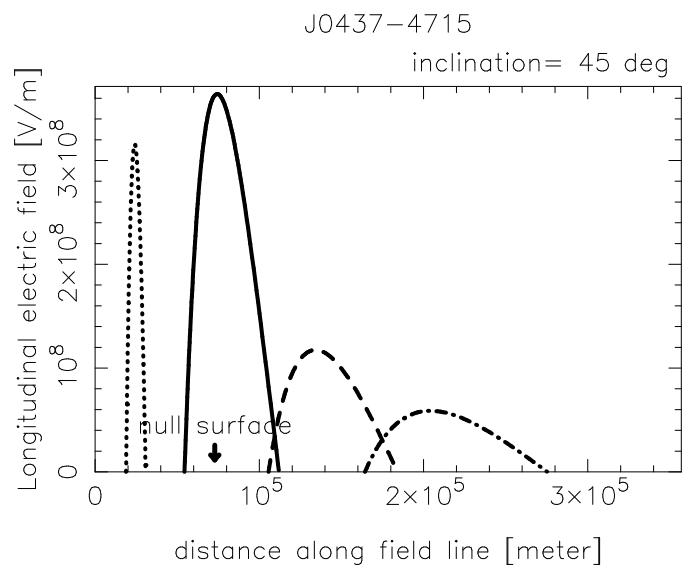

Figure 5. Distribution of the acceleration field for J0437-4715, a millisecond pulsar. The inclination angle is $45^{\circ}$. The abscissa, ordinate, and the lines are the same as figure figure 3 .

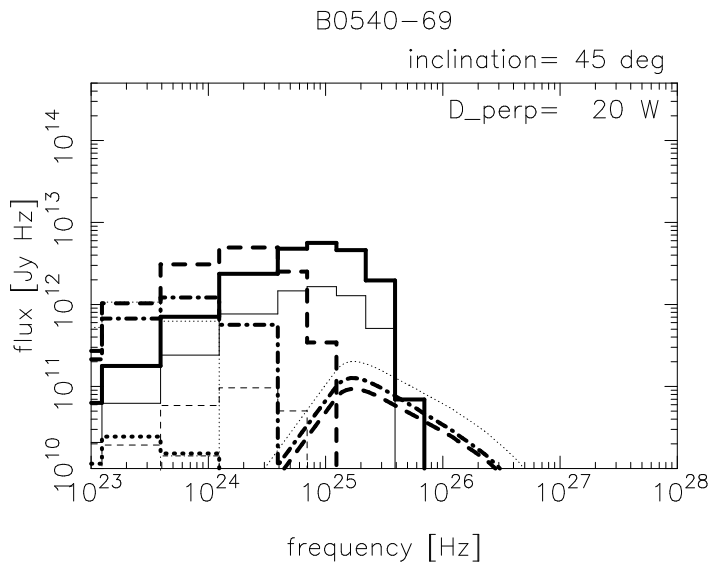

Figure 6. Pulsed gamma-ray spectra from B0540-69 magnetosphere when $\alpha_{\mathrm{i}}=45^{\circ}$. The thick and thin lines denote the spectra of outwardly and inwardly propagating $\gamma$-rays, respectively. The solid, dashed, dash-dotted, and dotted lines correspond to the cases $1,2,3$, and 4 , respectively (see text).

the case of another $\alpha_{\mathrm{i}}$ to compare the expected spectra with EGRET observations.

\subsubsection{General Features}

We first consider the general features of the $\gamma$-ray spectra. The GeV luminosity is the largest and hardest for case 1 $(\S 4.3)$, because both the potential drop and the maximum of $E_{\|}$is the largest. (see figs. 35 . It should be noted that the larger $\alpha_{\mathrm{i}}$ is, the harder the GeV spectrum becomes; this can be understood if we compare figures 12 and 13 , for instance. It should be noted that the same conclusion was derived independently for a vacuum gap in Paper V (e.g., $E_{\mathrm{c}}$ in table 3 ), in which the 'gap closure' condition was considered in stead of solving the stationary Vlasov equations $(\S$ 2). Physically speaking, this is because the distance of 


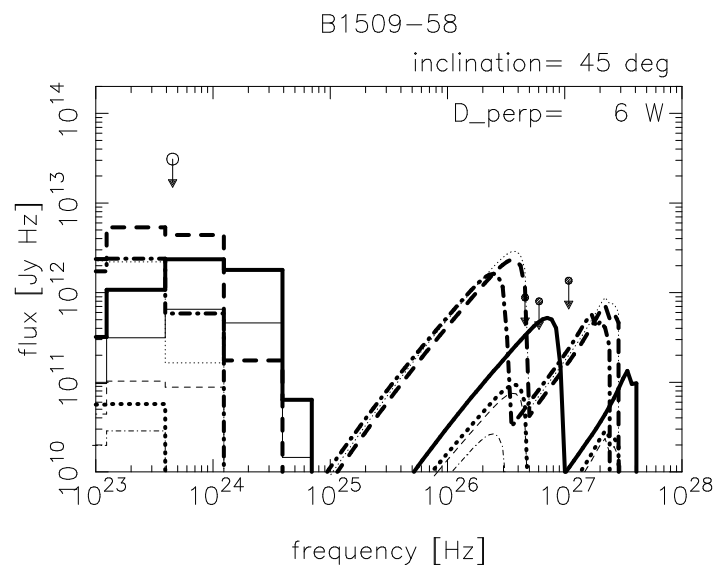

Figure 7. Pulsed gamma-ray spectra from B1509-58 when $\alpha_{\mathrm{i}}=$ $45^{\circ}$. The lines correspond to the same cases as in figure 7 .

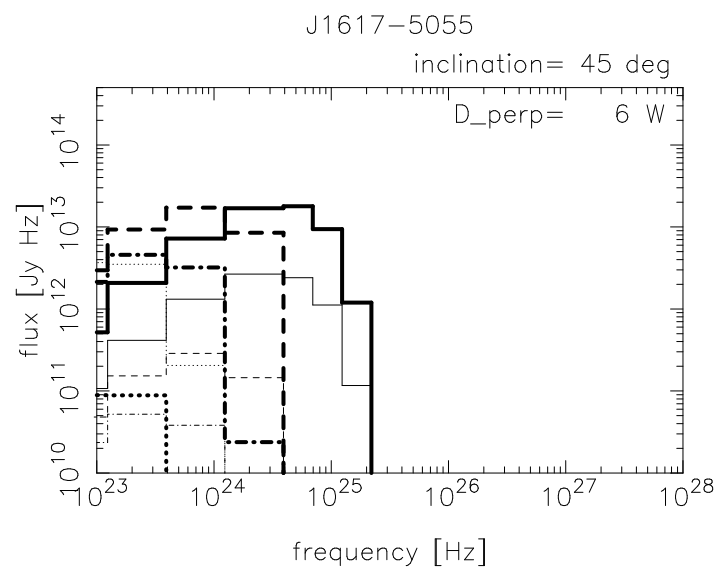

Figure 8. Pulsed gamma-ray spectra PSR J1617-30 when $\alpha_{i}=$ $45^{\circ}$. The lines correspond to the same cases as in figure 7 .

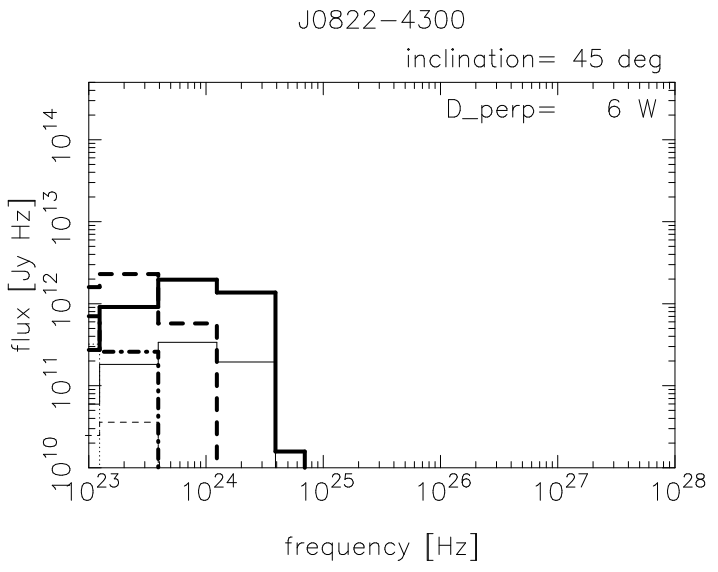

Figure 9. Pulsed gamma-ray spectra for the Vela pulsar when $\alpha_{\mathrm{i}}=45^{\circ}$. The lines correspond to the same cases as in figure 7 .

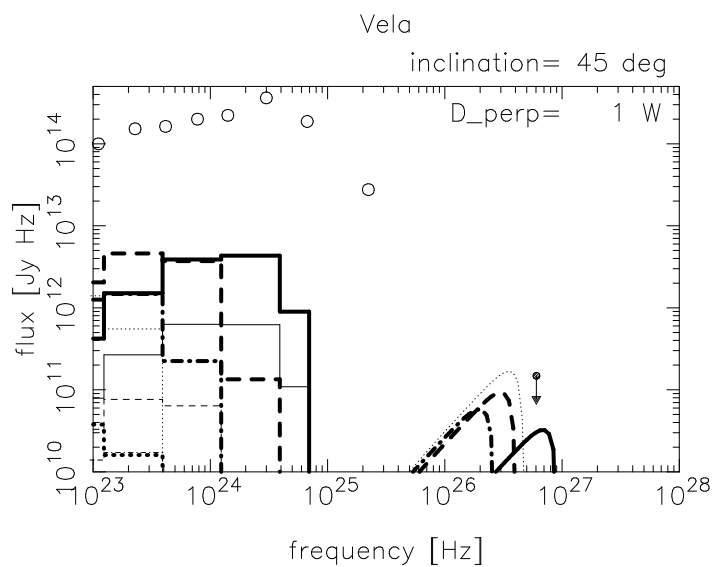

Figure 10. Pulsed gamma-ray spectra for the Vela pulsar when $\alpha_{\mathrm{i}}=45^{\circ}$. The lines correspond to the same cases as in figure 7 .

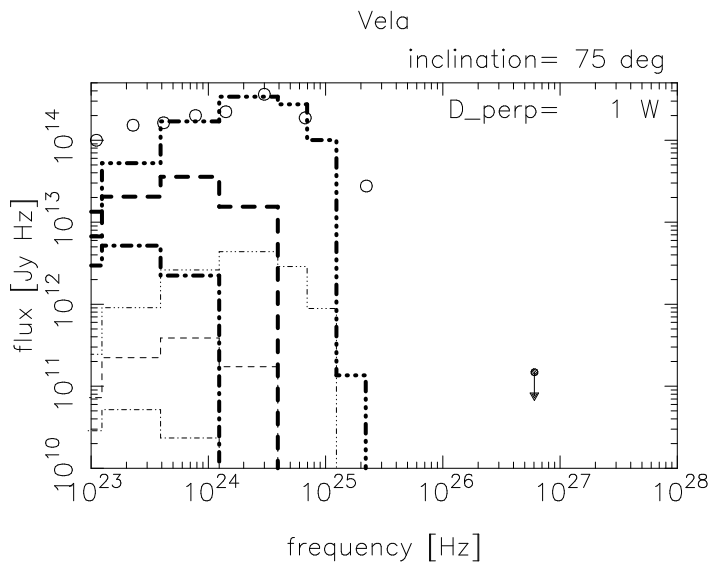

Figure 11. Pulsed gamma-ray spectra for the Vela pulsar when $\alpha_{\mathrm{i}}=75^{\circ}$. The dashed and dotted lines correspond to the same cases as in figure 7 , while the dash-dot-dot-dotted lines to $\left(j_{\text {gap }}, j_{1}, j_{2}\right)=(0.01,0.07,0)$.

the gap from the star decreases with increasing $\alpha_{\mathrm{i}}$ for the same set of $\left(j_{\text {gap }}, j_{1}, j_{2}\right)$. At small radii, the strong magnetic field enlarges $\rho_{\mathrm{GJ}}$, and hence $\left|d E_{\|} / d s\right|$, which results in a large $E_{\|}$at the gap center, especially when the gap is nearly vacuum (as the solid lines indicate in figs. 12 and 13 ).

We can also understand that the $\mathrm{TeV}$ fluxes are generally kept below the observational upper limits for appropriate $\mathrm{GeV}$ fluxes, or equivalently, appropriate cross sectional area, $D_{\perp}{ }^{2}$. Therefore, the problem of the excessive TeV flux does not arise for reasonable IR densities of individual pulsars. In the next subsection, we consider the expected spectra source by source.

\subsubsection{Comparison with EGRET observations}

First, we consider the Vela pulsar. It follows from the figures 10 and 11 that the pulsed $\mathrm{GeV}$ spectrum obtained by EGRET (Kanbach et al. 1994; Fierro et al. 1998) is impos- 


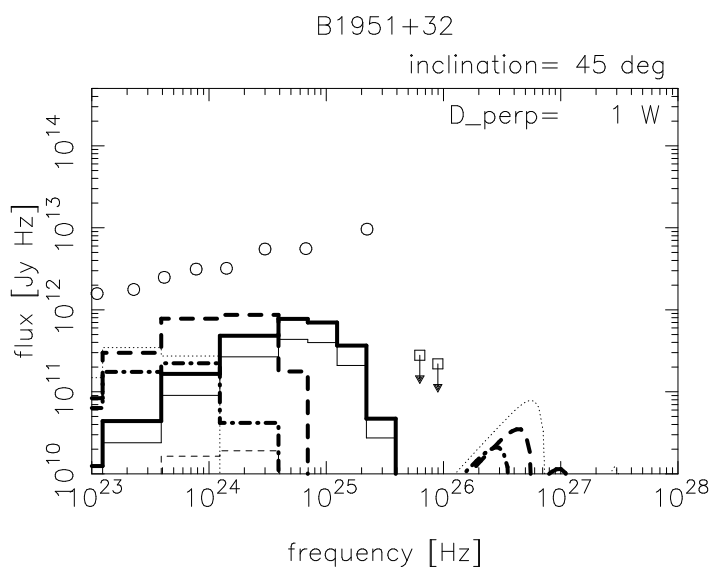

Figure 12. Pulsed gamma-ray spectra from B1951+32 when $\alpha_{\mathrm{i}}=45^{\circ}$. The lines correspond to the same cases as in figure 7 .

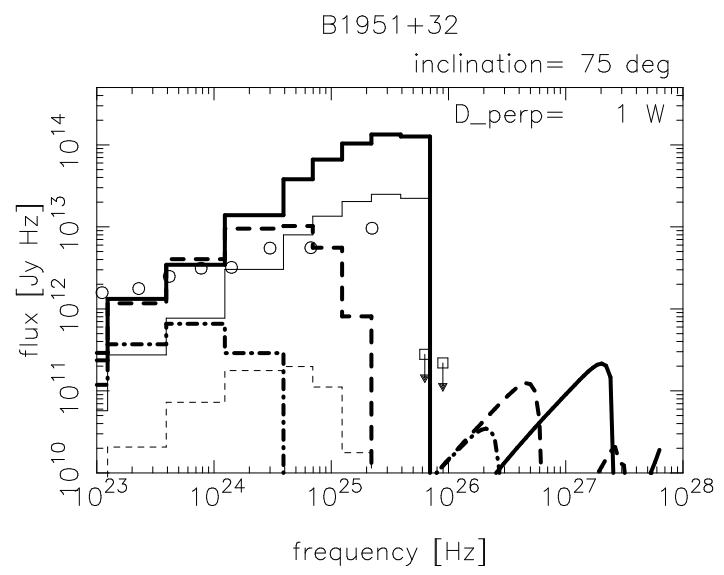

Figure 13. Pulsed gamma-ray spectra from B1951+32 when $\alpha_{\mathrm{i}}=75^{\circ}$. The lines correspond to the same cases as in figure 7 .

sible to be explained if the gap is located well inside of the null surface, as the dotted lines indicate. A large inclination angle and small current inflows are preferable to explain the observed flux around $10^{25} \mathrm{~Hz}$. For example, we present the case for $j_{1}=0.07$ and $j_{2}=0$ as the (thick and thin) dashdot-dot-dotted lines in figure 11. For $\alpha_{\mathrm{i}}>45^{\circ}$, expected TeV fluxes are well below the observed upper limit (Yoshikoshi et al. 1997).

Secondly, we consider B1951+32. It follows again that the observed $\mathrm{GeV}$ flux around $10^{25} \mathrm{~Hz}$ (Ramanamurthy et al. 1995) is impossible to be emitted if the gap is located well inside of the null surface, as the dotted lines indicate. Although the expected $\mathrm{TeV}$ flux is much less than the observed upper limits around $10^{26} \mathrm{~Hz}$ (Srinivanan et al. 1997), it may be detectable around $\nu \sim 10^{27} \mathrm{~Hz}$ with a future ground-based telescopes, if $\alpha_{\mathrm{i}}$ is as large as $75^{\circ}$.

Thirdly, we consider the Geminga pulsar. For this middle-aged pulsar, only the dotted lines are depicted in figures 14 and 15 , because there exist no solutions for cases 1 , 2, and 3. That is, for $\alpha_{\mathrm{i}}=45^{\circ}$ (or $60^{\circ}$ ), solutions exist only

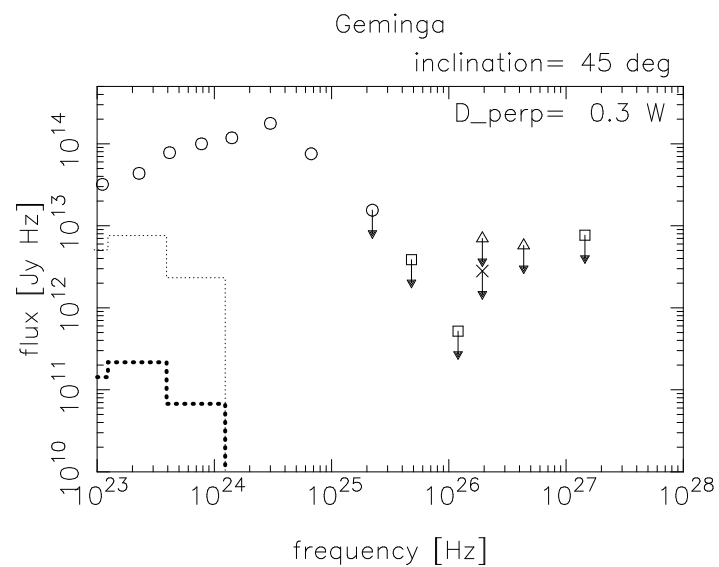

Figure 14. Pulsed gamma-ray spectra for the Geminga pulsar when $\alpha_{\mathrm{i}}=45^{\circ}$. The lines correspond to the same cases as in figure 7 .

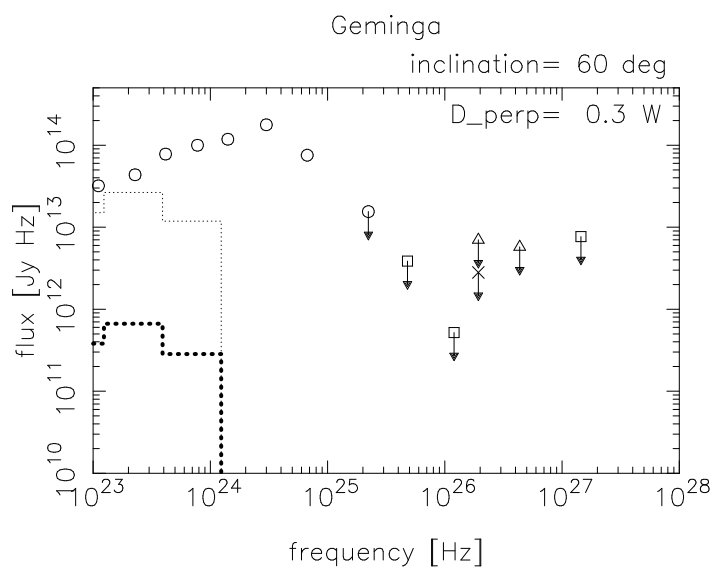

Figure 15. Pulsed gamma-ray spectra for the Geminga pulsar when $\alpha_{\mathrm{i}}=45^{\circ}$. The lines correspond to the same cases as in figure 7 .

when $j_{2}>0.073$ (or $j_{2}>0.017$ ), provided that $j_{\text {gap }}=0.01$ and $j_{1}=0$ hold. This is because its X-ray field is so weak that the gap extends towards the light cylinder and vanishes $d E_{\|} / d s$ at $\xi=\xi_{2}$ (i.e., forms a 'brim') at the critical value of $j_{2}$ ( $=0.073$ for $\alpha_{\mathrm{i}}=45^{\circ}$, say). Since the solutions exist in a limited region of the parameter space, the observed $\mathrm{GeV}$ spectrum above $10^{24} \mathrm{~Hz}$ (Mayer-Hasselwander et al. 1994) are impossible to be explained by the present theory. The $\mathrm{TeV}$ flux is expected to be much less than the observed upper limits (for pulsed upper limits, see Akerlof et al. 1993; for steady flux and/or its upper limits, see Weekes and Helmken 1977, Kaul et al. 1985, Vishwanath et al. 1993, Bowden et al. 1993).

Fourthly, we consider B1055-52. For this middle-aged pulsar, solutions exist in a wide parameter range, unlike the Geminga pulsar. However, we obtain too soft GeV spectra to fit the observations (Thompson et al. 1999). The difficulties 


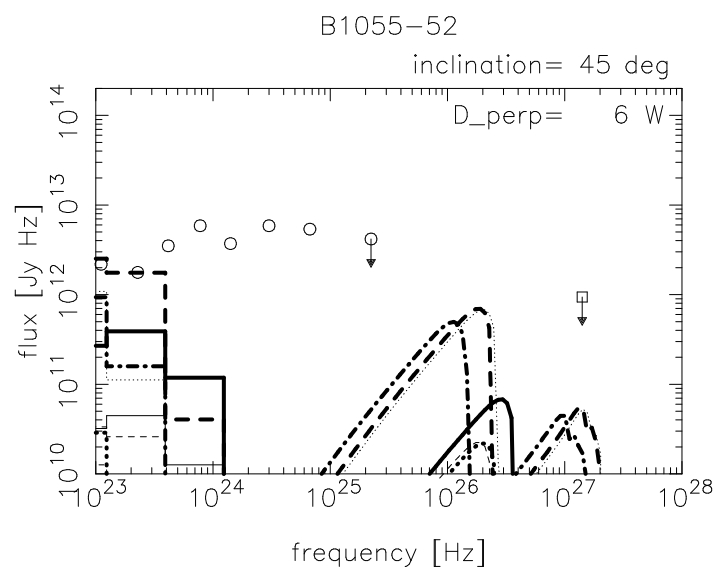

Figure 16. Pulsed gamma-ray spectra from B1055-52 when $\alpha_{\mathrm{i}}=45^{\circ}$. The lines correspond to the same cases as in figure 7 .

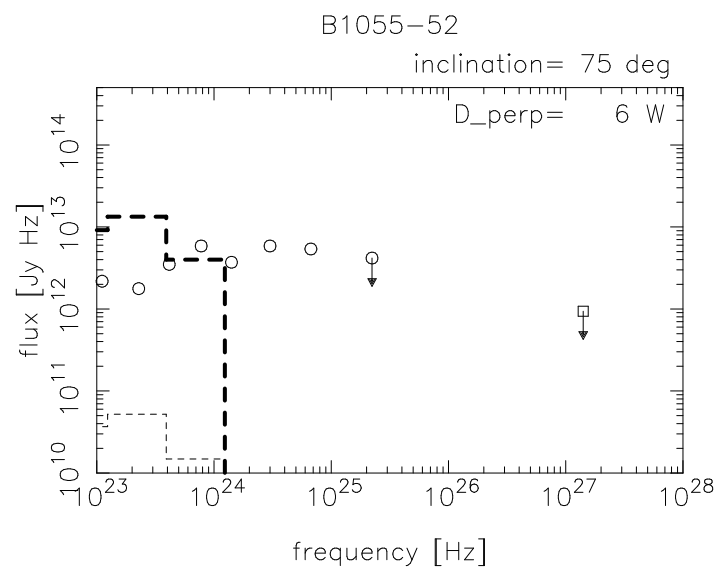

Figure 17. Pulsed gamma-ray spectra from B1055-52 when $\alpha_{\mathrm{i}}=75^{\circ}$. The lines correspond to the same cases as in figure 7 .

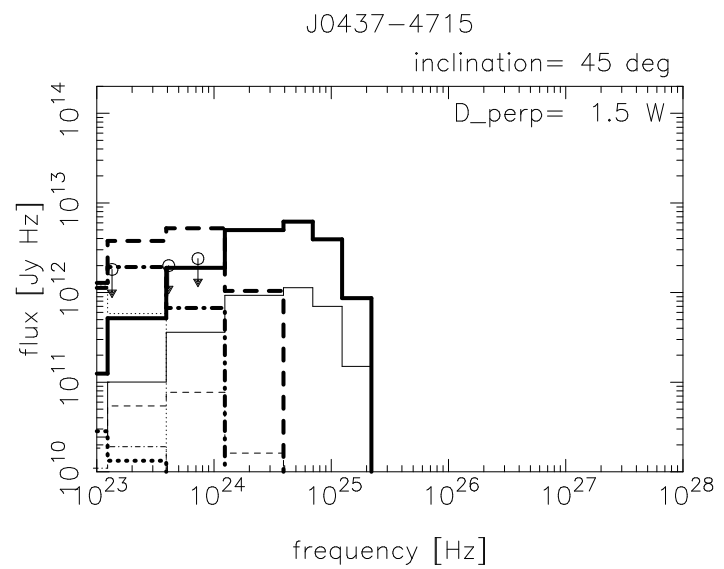

Figure 18. Pulsed gamma-ray spectra for J0437-4715 when $\alpha_{\mathrm{i}}=45^{\circ}$. The lines correspond to the same cases as in figure 7 .

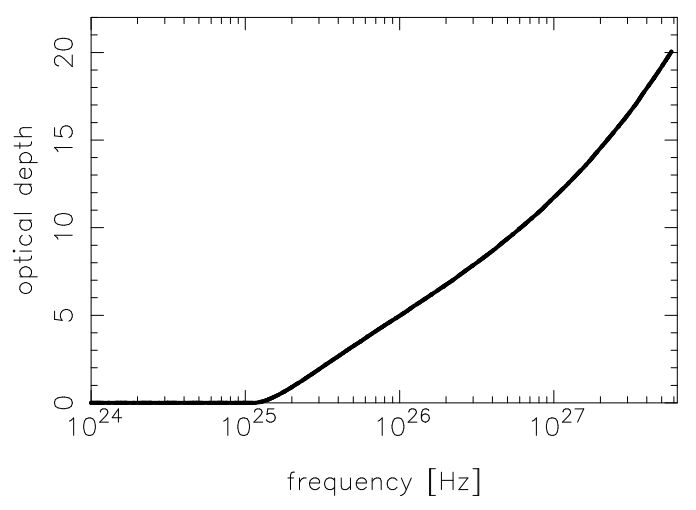

Figure 19. Pair production optical depth for a $\mathrm{TeV}$ photon to be absorbed in the homogeneous, isotropic IR field in the magnetosphere of pulsar B0540-69. The abscissa designates the $\gamma$-ray frequency.

in the application to these two middle-aged pulsars will be discussed in $\S 5.1$

Finally, we consider the other pulsars of which $\mathrm{GeV}$ fluxes were not detected by EGRET. For B0540-69, the basic properties are common with the Crab pulsar (Paper VII) as follows:

(1) $W \ll \varpi_{\text {LC }}$ holds because of its large X-ray density.

(2) Intrinsic $\mathrm{TeV}$ flux is comparable or even somewhat greater than the GeV flux but is significantly absorbed by its dense, magnetospheric IR field. For example, in case 1, the intrinsic TeV flux before absorption attains $1.7 \times 10^{13} \mathrm{Jy} \mathrm{Hz}$ at $1.4 \times 10^{27} \mathrm{~Hz}$, which is about two times greater than the maximum of the GeV flux. In figure 19, we present the absorption optical depth (eq. [56]). The resultant, absorbed $\mathrm{TeV}$ spectra are depited in figure 6, together with the $\mathrm{GeV}$ spectra.

(3) The curvature spectra are relatively hard (fig. 6), because its strong magnetic field at the gap increases $E_{\|}$, although its $W$ is small.

For B1509-58, its relatively less dense IR field (compared with Crab and B0540-69) cannot absorb enough TeV flux in the magnetosphere. As a result, the expected $\mathrm{TeV}$ flux becomes comparable with the observational upper limit while the $\mathrm{GeV}$ flux is well below the observational upper limits (fig. 7). As figures 12 and 13 indicate, the TeV flux does not increase so rapidly as $\mathrm{GeV}$ one with increasing $\alpha_{\mathrm{i}}$. We thus conclude that $\alpha_{\mathrm{i}}$ should be comparable with or greater than $45^{\circ}$ so that the $\mathrm{GeV}$ and $\mathrm{TeV}$ fluxes may be consistent with the observations. (Note that we can adjust the cross sectional area to decrease or increase the $\mathrm{GeV}$ and $\mathrm{TeV}$ fluxes at the same rate.)

In the case of J1617-5055, its GeV spectrum (fig. 8) is softer than Crab (Paper VII) and B0540-69, because its Xray field is less dense compared with these two young pulsars. Its $\mathrm{TeV}$ flux is small because of its small IR field assumed (§3.1).

The GeV spectrum of J0822-4300 (fig. 9) is softer than that of Vela, although the X-ray field more or less resembles each other. This is because the former's high-temperature 
surface emission provides a denser X-ray field, which shrinks the pair-production mean free path, and hence $W$ to reduce $E_{\|}$, compared with Vela.

For J0437-4715, the GeV flux is large (fig. 18), although we assume a small cross sectional area, $D_{\perp}{ }^{2}=(1.5 \mathrm{~W})^{2}$. This large Gev flux comes from the fact that millisecond pulsars have similar electrodynamic structure with young pulsars. Compared with the EGRET upper limit of pulsed emissions (Thompson 2000), we can expect that this millisecond pulsar is an attractive target for the next-generation $\mathrm{GeV}$ telescopes.

\section{DISCUSSION}

In summary, we have developed a one-dimensional model for an outer-gap accelerator in the magnetosphere of a rotating neutron star. When the positrons (or electrons) penetrate into the gap from the inner (or outer) boundary, the gap shifts outwards (or inwards). Applying the theory to Vela and $\mathrm{B} 1951+32$, we find that the GeV spectrum softens significantly and cannot explain the EGRET observations, if the gap is located well inside of the null surface. We can therefore conclude the gap should be located near to or outside of the null surface for these two pulsars. We also apply the theory to B0540-69, B1509-58, J1617-5055, J08224300, and J0437-4715 and find that the TeV fluxes are undetectable by current ground-based telescopes for moderate cross sectional areas of the gap for all the seven pulsars mentioned just above, if we estimate their magnetospheric IR field by interpolating radio and optical pulsed fluxes. For B0540-69, its intrinsically large $\mathrm{TeV}$ flux is absorbed by its dense IR field to become undetectable; this conclusion is analogous with what was obtained in Paper VII for the Crab pulsar.

\subsection{Difficulties in the Application to Middle-aged Pulsars}

We briefly discuss the difficulties in the application to middle-aged pulsars. As figures 1416 indicate, the fluxes from Geminga and B1055-52 above $10^{24} \mathrm{~Hz}$ are quite insufficient compared with EGRET observations. There are three reasons for this problem. First, special relativistic effects becomes important for the extented gaps of middle-aged pulsars, of which X-ray field is weak. For example, in equation (3), we neglected the terms containing higher orders in $(\Omega \varpi / c)^{2}$. Secondly, the global geometry of the magnetic field lines becomes important as as the gap extends. That is, the rectilinear approximation ( $\S 2.1$ cannot be applied to these two middle-aged pulsars. Thirdly, their large $E_{\|}$, which is a good fraction of the trans-field electric field, prevents particles from corotation (eq. [16]). On these grounds, applications of the present theory to middle-aged pulsars do not provide good agreement with observations.

\subsection{Gap Closure Condition}

In $\S 4.3 .1$, we pointed out that the potential drop decreases significantly as the gap shifts inwards from the null surface. In this subsection, we interpret this behavior from the gap closure condition investigated in Papers IV and V. In a stationary gap, the pair production optical depth, $W / \lambda_{\mathrm{p}}$, equals the ratio $N_{\gamma}\left(j_{\text {gap }} / j_{\text {tot }}\right)$, where $\lambda_{\mathrm{p}}$ and $N_{\gamma}$ refer to the pair production mean free path and the number of $\gamma$-rays emitted by a single particle, respectively. We thus obtain the following gap closure condition:

$W=\lambda_{\mathrm{p}} N_{\gamma} \frac{j_{\text {gap }}}{j_{\text {tot }}}$.

This condition is automatically satisfied by the stationarity of the Boltzmann equations. For example, consider the case when the external current is ten times greater than the created one in the gap. In this case, the expectation value for the $\gamma$-rays that are emitted from a single particle in the gap, should be one tenth so that a stationary pair production cascade may be maintained, because not only the produced particles but also the externally injected particles emit $\gamma$ rays in the same way.

As the gap shifts inwards, the X-ray density increases to reduce $\lambda_{\mathrm{p}}$. At the same time, the ratio $j_{\text {gap }} / j_{\text {tot }}$ decreases as $j_{2}$ increases. As a result, $W$ decreases very rapidly with increasing $j_{2}$. Owing to the rapidly decreasing $W$, the integrated potential drop significantly decreases, although the local $\rho_{\mathrm{GJ}}$, and hence $\left|d E_{\|} / d s\right|$ increases inwards.

If the gap shifts outwards, on the other hand, the increase of $\lambda_{\mathrm{p}}$ and the decrease of $j_{\text {gap }} / j_{\text {tot }}$ partially cancel each other. As a result, the potential drop decreases only slightly with increasing $j_{1}$.

\subsection{Comparison with ZC97}

It is worth comparing the present method with ZC97, who onsidered that the gap size is limited so that the emitted $\gamma$-rays may have energies just above the pair production threshold in collisions with the surface X-rays that are due to the bombardment of produced particles in the gap. Their method qualitatively agrees with the current gap closure condition (eq. 66]), provided $j_{\text {gap }} / j_{\text {tot }} \sim 1$ (see $\S 5.8$ in Paper $\mathrm{V})$; case $1\left(j_{1}=j_{2}=0\right)$ corresponds to this case. However, as $j_{2}$ increases, the factor $\left(j_{\text {gap }} / j_{\text {tot }}\right)$ in equation 66 becomes small to reduce $W$. In other words, the closure condition adopted by ZC97 would become inconsistent with stationary Vlasov equations ( $\S 2$ ), if we were to overextraporate it to the case when $j_{2} \sim 1$. In the same manner, we cannot overextraporate the gap-closure condition of ZC97 to the case when $j_{1}$ increases and hence the gap shifts towards the light cylinder (as cases 2 and 3 ), because $j_{\text {gap }} / j_{\text {tot }}$ decreases substantially.

\subsection{Inverse Compton Scatterings in Young Pulsar Magnetosphere}

Although the dense IR field of young pulsars (B0540-69, B1509-58) results in a relatively large intrinsic $\mathrm{TeV}$ emission compared with other pulsars, the intrinsic $\mathrm{TeV}$ flux is not more than the GeV flux, provided $\alpha_{\mathrm{i}}>45^{\circ}$. Therefore, the radiation reaction force is caused primarily by the curvature process rather than the inverse Compton scatterings, as long as $j_{2}$ is less than 0.3 . However, if $j_{2}$ exceeds 0.3 , we cannot in general rule out the possibility of the case when the radiation reaction is caused by IC scatterings. This is because the dense X-ray field will suppress the particle Lorentz factors 
(Paper II) below $10^{7}$, and because such less-energetic particles scatter copious IR photons into lower $\gamma$-ray energies with large cross sections $\left(\sim \sigma_{\mathrm{T}}\right)$. As a result, the $\mathrm{GeV}$ emissions may be mainly due to IC scattering in this case. Moreover, since the magnetic field becomes very strong close to the star, not only the synchro-curvature process (ZC97), but also a magnetic pair production becomes important. There is room for further investigation of the case when an 'outer' gap is located close to the polar cap.

\section{ACKNOWLEDGMENTS}

One of the authors (K. H.) wishes to express his gratitude to Drs. Y. Saito and A. Harding for valuable advice. He also thanks the Astronomical Data Analysis Center of National Astronomical Observatory, Japan for the use of workstations.

\section{REFERENCES}

Akerlof, C. W. et al. 1993, A\& A 274, L17

Aschenbach, B., Brinkmann, W. 1975, A \& A 41, 147

Becker, W., Trümper, J. 1993, Nature 365, 528

Berestetskii, V. B., Lifshitz, E. M. \& Pitaevskii, L. P., 1989, Quantum Electrodynamics 3rd ed.

Beskin, V. S., Istomin, Ya. N., Par'ev, V. I. 1992, Sov. Astron. $36(6), 642$

Blumenthal, G. R., Gould, R. J. 1970, Rev. Mod. Phys., 42, 237 owden

Bowden, C. C. G. et al. 1993, Phys. G.: Nucl. Part. Phys. 19, L29

Caraveo, P. A., Mereghetti, S., Bignami, G. F. 1994, ApJ 423, L125

Caraveo, P. A., Bignami, G. F., Mignani, R., Taff, L. G. 1996, ApJ 461, L91

Cheng, K. S. 1994 in Towards a Major Atmospheric Cerenkov Detector III, Universal Academy Press, Inc., p. 25

Cheng, K. S., Ho, C., Ruderman, M., 1986a ApJ, 300, 500

Cheng, K. S., Ho, C., Ruderman, M., 1986b ApJ, 300, 522

Chiang, J., Romani, R. W. 1992, ApJ, 400, 629

Chiang, J., Romani, R. W. 1994, ApJ, 436, 754

Daishido, T. 1975, PASJ 27, 181

Daugherty, J. K., Harding, A. K. 1982, ApJ, 252, 337

Daugherty, J. K., Harding, A. K. 1996, ApJ, 458, 278

Dermer, C. D., Sturner, S. J. 1994, ApJ, 420, L75

Downs, G. S., Reichley, P. E., Morris, G. A. 1973, ApJ 181, L143

Edwards, P. G., et al. 1994 A\& A 291, 468

El-Gowhari, A., Arponen, J. 1972, Nuovo Cimento, 11B, 201

Fierro, J. M., Michelson, P. F., Nolan, P. L., Thompson, D. J., 1998, ApJ 494, 734

Gil, J. A., Krawczyk, A. 1996 MNRAS 285, 566

Greenstein, G., Hartke, G. J. 1983, ApJ 271, 283

Greiveldinger, C., Camerini, U., Fry, W., Markwardt, C. B., Ögelman, H., Safi-Harb, S., Finley, J. P., Tsuruta, S. 1996, ApJ 465, L35

Halpern, J. P., Martin, C., and Marshall, H. L., 1996 ApJ 462, 908

Halpern, J. P. Wang, Y. H., 1997, ApJ 477, 905

Hardee, P. E., Rose, W. K. 1974, ApJ 194, L35

Harding, A. K., Tademaru, E., Esposito, L. S. 1978, ApJ, 225, 226

Hirotani, K. 2000a, MNRAS 317, 225 (Paper IV)

Hirotani, K. 2000b ApJ 545, in press (Paper V)

Hirotani, K. 2000c PASJ 52, 1 (Paper VI)

Hirotani, K. Okamoto, I., 1998, ApJ, 497, 563
Hirotani, K. Shibata, S., 1999a, MNRAS 308, 54 (Paper I) Hirotani, K. Shibata, S., 1999b, MNRAS 308, 67 (Paper II) Hirotani, K. Shibata, S., 1999c, PASJ 51, 683 (Paper III)

Hirotani, K. Shibata, S., 2000, submitted to ApJL (Paper VII)

Kanbach, G., Arzoumanian, Z., Bertsch, D. L., Brazier, K. T. S., Chiang, J., Fichtel, C. E., Fierro, J. M., Hartman, R. C., et al. 1994, A \& A 289, 855

Kaspi, V. M., Lackey, J. R., Manchester, R. N., Bailes, M., Pace, R. 2000, ApJ 528, 445

Kaul, R. K. et al. 1985, Proc. 19th ICRC (La Jalla), 1, 165

Kawai, N., Okayasu, R., Sekimoto, Y. 1993, in Compton GammaRay Observatory, ed. M. Friedlander, N. Gehrels, D. J. Macomb (AIP Conf. Proc. 280) (New York; AIP), 213

Kifune, T. 1996, Space Science Reviews 75, 31

Krause-Polstorff, J., Michel, F. C. 1985a MNRAS 213, 43

Krause-Polstorff, J., Michel, F. C. 1985b A\& A, 144, 72

Kundt, W., Schaaf, R. 1993, Ap \& SpSc, 200, 251

Manchester, R. N., Wallace, P. T., Peterson, B. A., Elliott, K. H. 1980, MNRAS 190, 9p

Manchester, R. N., Mar, D. P., Lyne, A. G., Kaspi, V. M., Johnston, S. 1993, ApJ 403, L29

Mayer-Hasselwander, H. A., Bertsch, D. L., Brazier, T. S., Chiang, J., Fichtel, C. E., Fierro, J. M., Hartman, R. C., Hunter, S. D. 1994, ApJ 421, 276

Middleditch, J., Pennypacker, C. 1985, Nature 313, 659

Mignani, R., Caraveo, P. A., Bignami, G. F. 1997, ApJ 474, L51

Nel, H. I., De Jager, O. C., Raubenheimer, B. C., Brink, C., Meintjes, P. J., Nortt, A. R. 1993, ApJ 418, 836

Nolan, P. L., Arzoumanian, Z., Bertsch, D. L., Chiang, J., Fichtel, C. E., Fierro, J. M., Hartman, R. C., Hunter, S. D., et al. 1993, ApJ 409, 697

Ochelkov, Yu. P., Usov, V. V. 1980a, Ap. Space Sci., 69, 439

Ochelkov, Yu. P., Usov, V. V. 1980b, Sov. Astr. Letters (USA), 6,414

Ögelman, H., Finley, J. P., Zimmermann, H. U. 1993, Nature 361, 136

Pavlov, G. G., Shibanov, Yu. A., Ventura, J., Zavlin, V. E. 1994, A \& A 1994, 289, 837

Petre, R., Becker, C. M., Winkler, P. F. 1996, ApJ 465, L43

Ramanamurthy, P. V., Bertsch, D. L., Dingus, L., Esposito, J. A., Fierro, J. M., Fichtel, C. E., Hunter, S. D., Kanbach, G., et al. 1995, ApJ 447, L109

Reynoso, E. M., Dubner, G. M., Goss, W. M., Arnal, E. M. 1995, AJ 110,318

Romani, R. W. 1987, ApJ, 313, 718

Romani, R. W. 1996, ApJ, 470, 469

Romani, R. W., Yadigaroglu, I. A. 1995, ApJ 438, 314

Rybicki, G. B. \& Lightman, A. P., 1979, Radiative processes in astrophysics, (John Wiley \& Sons, New York)

Safi-Harb, S. Ögelman, H., Finley, J. P. 1995 1995, ApJ 439, 722

Saito, Y. 1998, Ph.D. Thesis

Sandhu, J. S., Bailes, M.m Manchester, R. N., Navarro, J. Kulkarni, S., et at., 1997, ApJ 478, L95

Shearer, A. et al. 1998, A \& A 335, L21

Seward, F. D., Harnden, F. R., Szymkowiak, A., Swank, J. 1984, ApJ 281, 650

Shibanov, Yu. A., Zavlin, V. E., Pavlov, G. G., Ventura, J. 1992 A \& A 266, 313

Shibata, S. 1995, MNRAS 276, 537

Shibata, S., Miyazaki, J., Takahara, F. 1998, MNRAS 295, L53

Shibata, S., Hirotani, K. 2000, in preparation

Srinivanan, R. et al. 1997, ApJ 489, 170

Sturner, S. J., Dermer, C. D., Michel, F. C. 1995, ApJ 445, 736

Taylor, J. H., Manchester, R. N., Lyne, A. G. 1993, ApJS 88, 529

Thompson, D. J., Bailes, M., Bertsch, D. L., Esposito, J. A., Fichtel, C. E., Harding, A. K., Hartman, R. C., Hunter, S. D. 1996, ApJ 465, 385 
Thompson, D. J., Bailes, M., Bertsch, D. L., Cordes, J., D'Amico, N., Esposito, J. A., Finley, J., Hartman, R. C., et al. 1999, ApJ 516, 297

Thompson, D. J. 2000, Adv. Space Res. 25, 659

Torii, K., Kinugasa, K., Toneri, T., Asanuma, T., Tsunemi, H., Dotani, T., Mituda, K., Gotthelf, E. V., Petre, R. 1998, ApJ 494, L207

Usov, V. V. 1994, ApJ 427, 394

Vishwanath, P. R. et al. 1993, A \& A 267, L5

Weekes, T. C., Helmken, H. F. 1977, Proc. 12th ESLAB Symp., 124,39

Yoshikoshi, T., Kifune, T., Dazeley, S. A., Edwards, P. G., Hara, T., Hayami, Y., Kakimoto, F., Konishi, T. 1997, ApJ 487, L65

Zavlin, V. E., Pavlov, G. G., Shibanov, Yu. A., Ventura, J. 1995, A\& A, 297, 441

Zavlin, V. E., Shibanov, Yu. A., Pavlov, G. G. 1995, Astron. Letters, 21, 149

Zavlin, V. E., Pavlov, G. G., 1998, A\& A, 329, 583

Zhang, L. Cheng, K. S. 1997, ApJ 487, 370 (ZC97) 